University of Louisville

ThinkIR: The University of Louisville's Institutional Repository

\title{
3D-reconstruction of human jaw from a single image : integration between statistical shape from shading and shape from shading.
}

Mohamad Ghanoum 1990-

University of Louisville

Follow this and additional works at: https://ir.library.louisville.edu/etd

Part of the Electrical and Computer Engineering Commons

\section{Recommended Citation}

Ghanoum, Mohamad 1990-, "3D-reconstruction of human jaw from a single image : integration between statistical shape from shading and shape from shading." (2015). Electronic Theses and Dissertations. Paper 2154.

https://doi.org/10.18297/etd/2154

This Master's Thesis is brought to you for free and open access by ThinkIR: The University of Louisville's Institutional Repository. It has been accepted for inclusion in Electronic Theses and Dissertations by an authorized administrator of ThinkIR: The University of Louisville's Institutional Repository. This title appears here courtesy of the author, who has retained all other copyrights. For more information, please contact thinkir@louisville.edu. 


\title{
3D-RECONSTRUCTION OF HUMAN JAW FROM A SINGLE IMAGE: INTEGRATION BETWEEN STATISTICAL SHAPE FROM SHADING AND SHAPE FROM SHADING
}

\author{
By \\ Mohamad Ghanoum \\ B.S. (ECE), University of Kalamoon, 2013 \\ A Thesis \\ Submitted to the Faculty of the \\ University of Louisville \\ J. B. Speed School of Engineering \\ as Partial Fulfillment of the Requirements \\ for the Degree of \\ Master of Science in Electrical Engineering \\ Department of Electrical and Computer Engineering \\ University of Louisville \\ Louisville, Kentucky
}

May 2015 
Copyright 2015 by Mohamad Ghanoum

All rights reserved 



\title{
3D-RECONSTRUCTION OF HUMAN JAW FROM A SINGLE IMAGE: INTEGRATION BETWEEN STATISTICAL SHAPE FROM SHADING AND SHAPE FROM SHADING
}

\author{
By \\ Mohamad Ghanoum \\ B.S. (ECE), University of Kalamoon, 2013
}

\begin{abstract}
A Thesis Approved on
April 22, 2015

by the Following Reading and Examination Committee:
\end{abstract}

Thesis Director, Dr. Aly A. Farag

Dr. John F. Naber

Dr. Bruce Alphenaar

Dr. Olfa Nasraoui 


\section{DEDICATION}

I would like to dedicate this thesis to my parents, my uncle Dr. kheder Kutmah , and my aunt Fatema Zuhayli . 


\section{ACKNOWLEDGEMENTS}

I would like to express my deepest gratitude to my advisor Dr. Aly A. Farag for his guidance, support, and encouragement to pursue a Master degree on the computer vision and medical imaging field.

I would like to acknowledge the other members of my Master committee Dr. John F. Naber, Dr. Bruce Alphenaar and Dr. Olfa Nasraoui, for spending time and effort in reading and reviewing my work.

I would like to thank especially Dr. Ali Mahmoud and Eslam Mostafa for their invaluable guidance and support in research. Without those two, I could not have done any of this.

I would like to thank Dr. Khaled Katmeh and Dr. Zena Sakka for their assistance in the terminology of dentistry.

Also, I would also like to thank Mr. Mike Miller and all CVIP Lab members for their friendship and assistance. I am especially grateful to Dr. Ahmed Shalaby and Dr.Amal Farag.

Also, words cannot describe how I am indebted to my Parents, my uncle Dr. Kheder

Kutmah, and my aunt Fatema Zuhayli, for their pain, suffering and sacrifices made during the journey of this study.

Finally, I would like to thank my cousin Dr. Ahmed Kutmah. 


\begin{abstract}
3D-RECONSTRUCTION OF HUMAN JAW FROM A SINGLE IMAGE: INTEGRATION BETWEEN STATISTICAL SHAPE FROM SHADING AND SHAPE FROM SHADING
\end{abstract}

\author{
Mohamad Ghanoum
}

April 22, 2015

Object modeling is a fundamental problem in engineering, involving talents from computer-aided design, computational geometry, computer vision and advanced manufacturing. The process of object modeling takes three stages: sensing, representation, and analysis. Various sensors may be used to capture information about objects; optical cameras and laser scanners are common with rigid objects, while X-ray, CT and MRI are common with biological organs. These sensors may provide a direct or indirect inference about the object, requiring a geometric representation in the computer that is suitable for subsequent usage. Geometric representations that are compact, i.e., capture the main features of the objects with minimal number of data points or vertices, fall into the domain of computational geometry. Once a compact object representation is in the computer, various analysis steps can be conducted, including recognition, coding, 
transmission, etc. The subject matter of this thesis is object reconstruction from a sequence of optical images. An approach to estimate the depth of the visible portion of the human teeth from intraoral cameras has been developed, extending the classical shape from shading (SFS) solution to non-Lambertian surfaces with known object illumination characteristics. To augment the visible portion, and in order to have the entire jaw reconstructed without the use of CT or MRI or even X-rays, additional information will be added to database of human jaws. This database has been constructed from an adult population with variations in teeth size, degradation and alignments. The database contains both shape and albedo information for the population. Using this database, a novel statistical shape from shading (SSFS) approach has been created.

To obtain accurate result from shape from shading and statistical shape from shading, final step will be integrated two approaches (SFS,SSFS) by using Iterative Closest Point algorithm (ICP).

Keywords: computer vision, shading, 3D shape reconstruction, shape from shading, statistical, shape from shading, Iterative Closest Point. 


\section{TABLE OF CONTENTS}

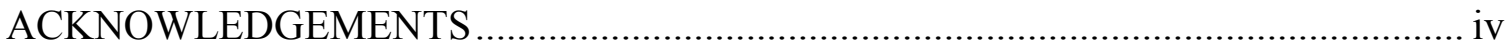

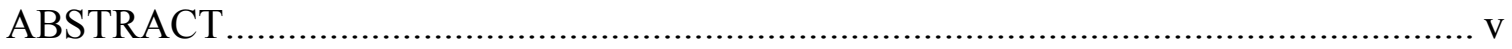

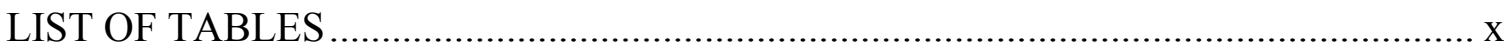

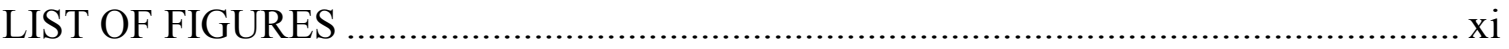

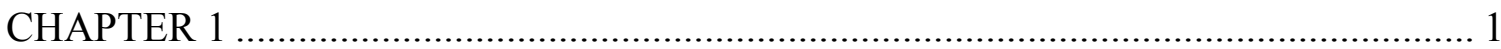

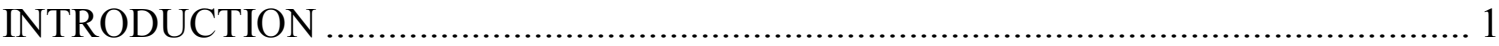

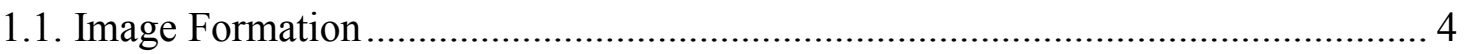

1.1.1. Geometric camera models ............................................................................ 5

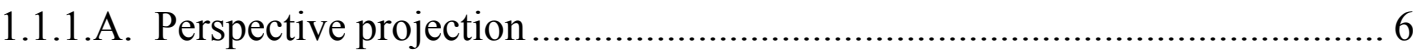

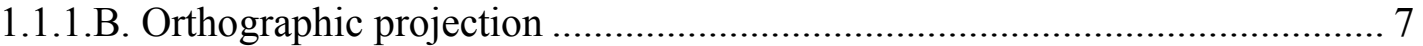

1.2. Shape From Shading VS Statistical Shape From Shading .................................. 8

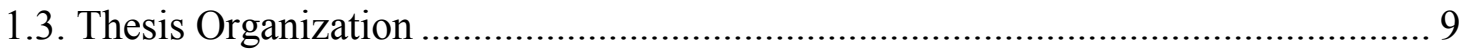

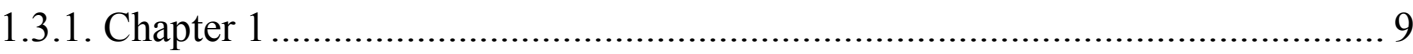

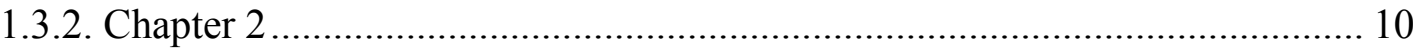

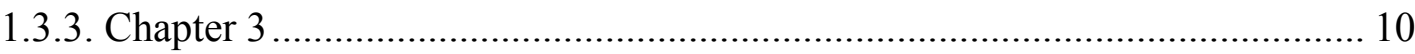

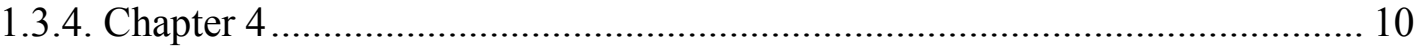

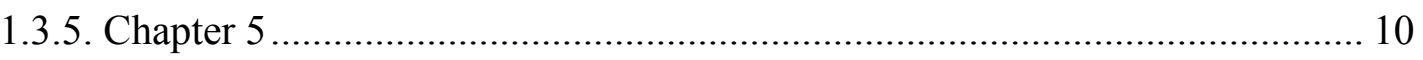

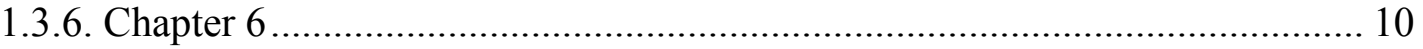

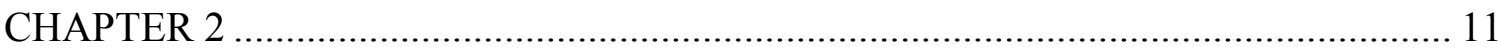

3D RECONSTRUCTION OF THE HUMAN JAW USING STATISTICAL SHAPE

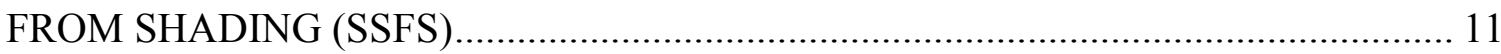

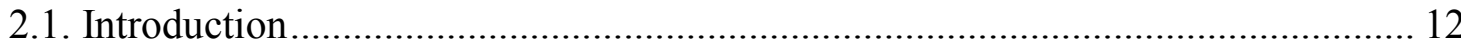

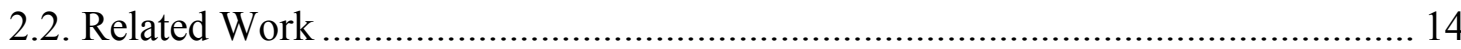

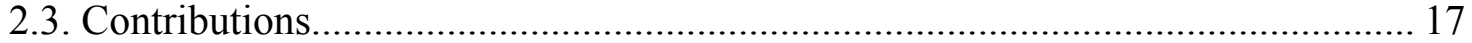




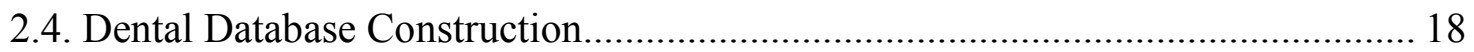

2.5. Anatomical Jaw Landmarks - Proposed Definition ............................................... 19

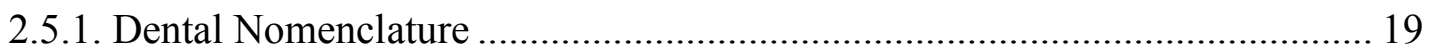

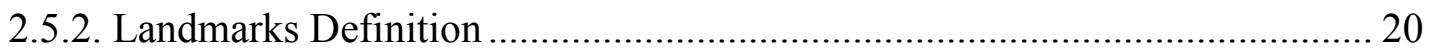

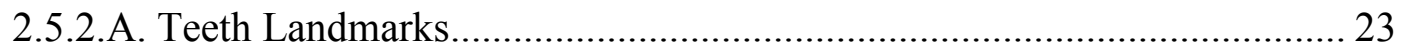

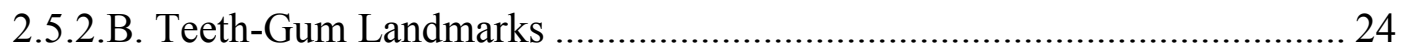

2.5.3. Landmark Localization in Optical Images.................................................... 25

2.6. Illumination-Invariant Statistical Shape from Shading......................................... 27

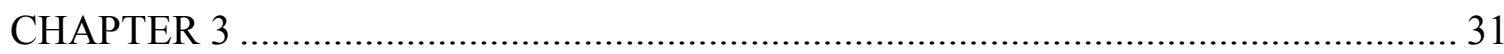

3D RECONSTRUCTION OF THE HUMAN TEETH USING SHAPE FROM

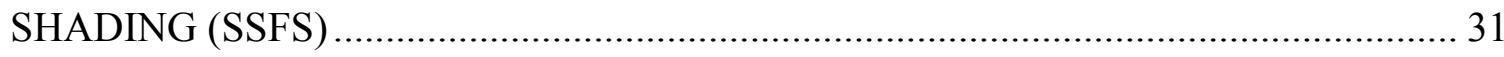

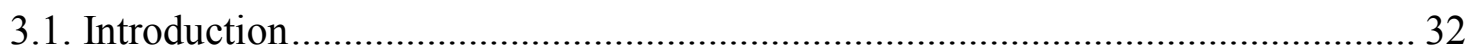

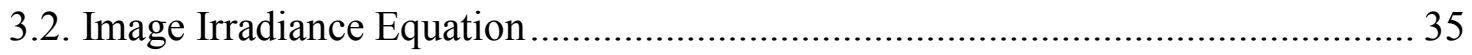

3.3. Parametric SFS for Non-Lambertian Surfaces …………………………............. 36

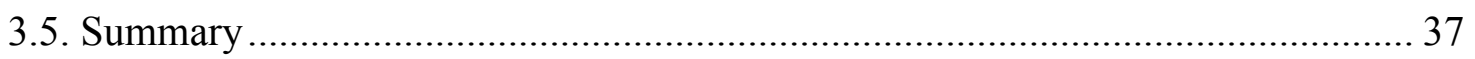

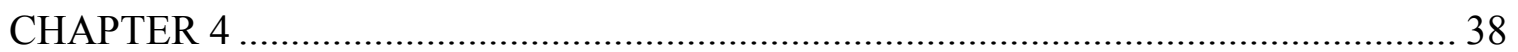

ITERATIVE CLOSEST POINT TECHNIQUE FOR 3D SHAPE REGISTRATION .... 38

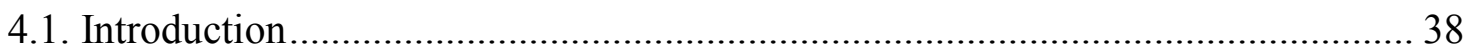

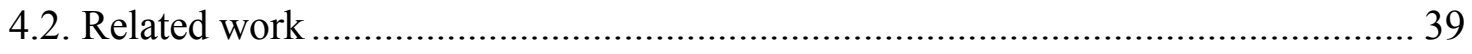

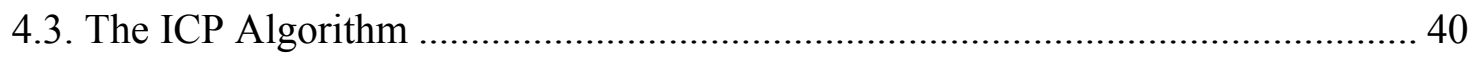

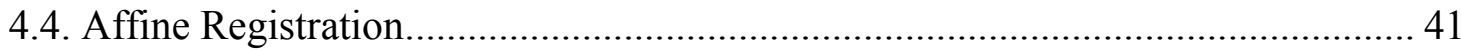

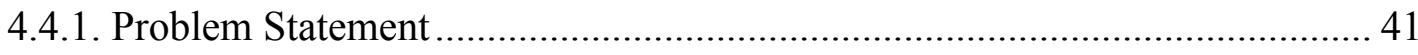

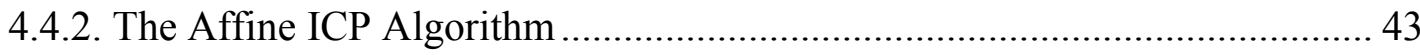

4.5. Landmarks definition for Iterative Closest Point .................................................... 44

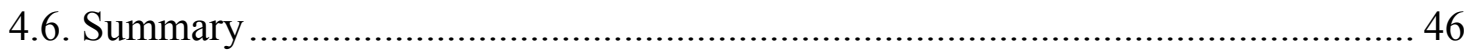

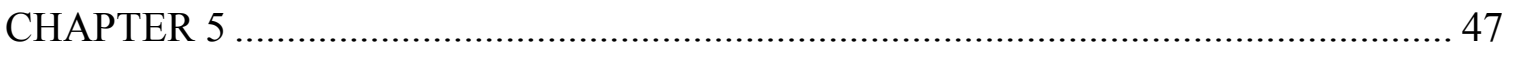

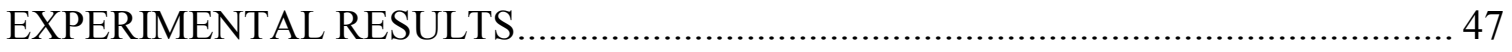

5.1. 3D Reconstruction of Human Jaw using Statistical Shape from Shading (SSFS) 47

5.1.1. Visible Crowns Reconstruction ................................................................... 48 
5.2. 3D Reconstruction of Human Teeth from a Single Image using Shape from

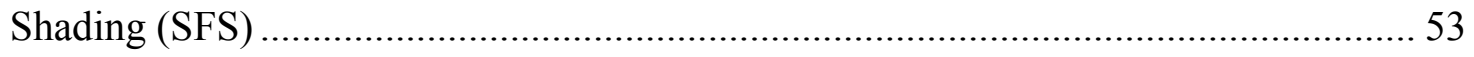

5.3. Iterative Closest Point Technique for 3D Shapes Registration.............................. 57

5.4. Graphic User Interface Approach for Jaw Construction Shape ............................. 64

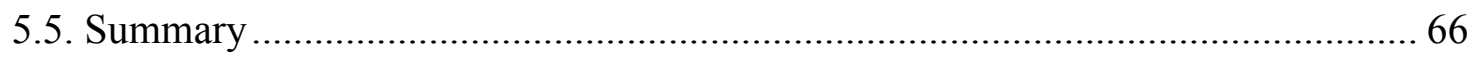

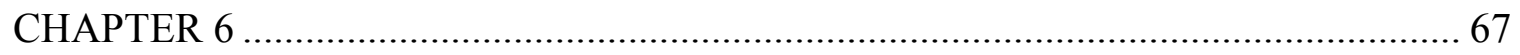

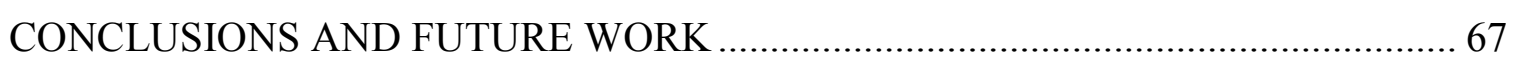

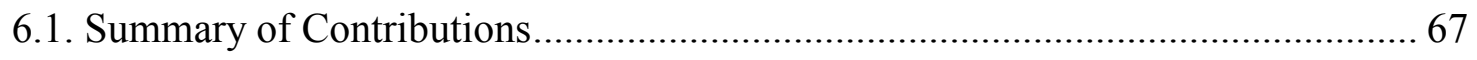

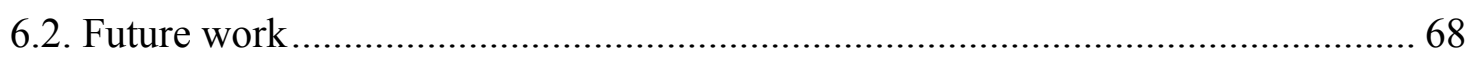

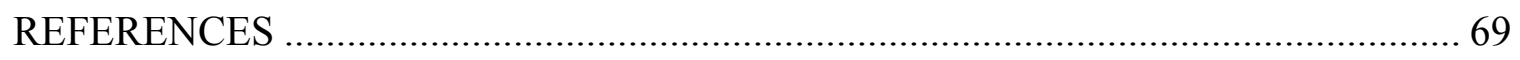

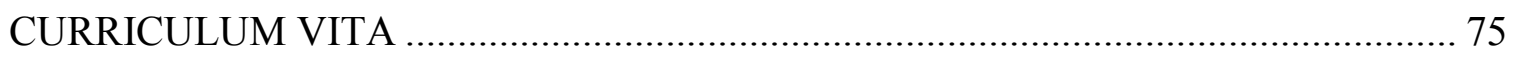




\section{LIST OF TABLES}

Table 2.1. Database construction of the human Jaw: subjects categorized with respect to

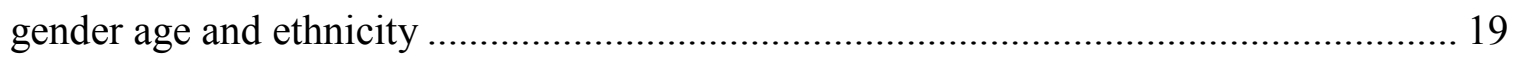

Table 5.1. Average and Std of the whole jaw surface reconstruction accuracy (RMS) in

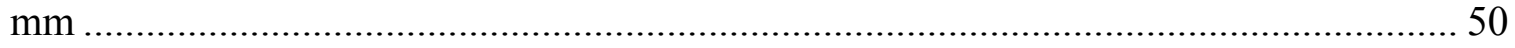

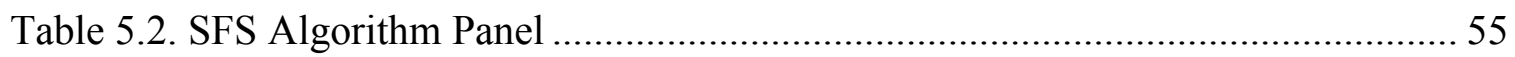

Table 5.3. Overall surface reconstruction accuracy (RMS) in $\mathrm{mm}$............................... 55 


\section{LIST OF FIGURES}

Figure 1.1. A pinhole camera produces an image that is a perspective projection of the world.

Figure 1.2. The geometry of the perspective projection. The point $\mathrm{P}=(\mathrm{X}, \mathrm{Y}, \mathrm{Z})$ is

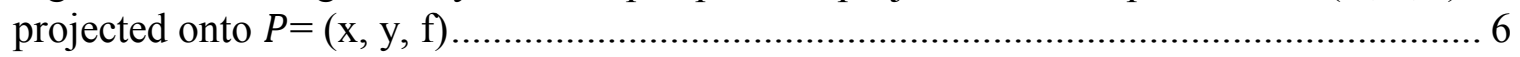

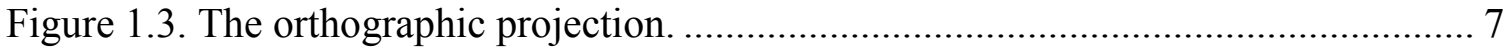

Figure 2.1. Block diagram of the proposed model-based human jaw shape recovery: (a) An aligned ensemble of the shapes and textures (oral cavity images) of human jaws is used to build the 3D shape model. (b) Given the texture and surface normals (defining the shape) of a certain jaw in the ensemble, harmonic basis images are constructed. Given an input oral cavity image under general unknown illumination and a set of human jaw anatomical landmark points: (c) Dense correspondence is established between the input image and each jaw sample in the ensemble, where each pixel position within the convex hull of a reference jaw shape corresponds to a certain point on a sample jaw (shape and texture) and in the same time to a certain point on the input image. (d) The input image, in the reference frame, is projected onto the subspace spanned by the harmonic basis of each sample in the ensemble which are scaled (using the projection coefficients) and summed-up to construct the harmonic projection (HP) images which encodes the illumination conditions of the input image. Such images are then used to construct an HP model of the input image. (e) The inherit relation between the HP images and the corresponding shape is cast as a regression framework where partial least squares is used to solve for shape coefficients to recover the shape of the input image.

Figure 2.2. Sample of the human jaw (pre-repair) lower and upper jaws: first column shows the 2D images, 3D scans using cone beam CT machine shows in the second column..... 21

Figure 2.3. Sample of the human jaw (post-repair ) lower and upper jaws: first column shows the 2D images, 3D scans using cone beam CT machine shows in the second column. 22

Figure 2.4. Dental nomenclature, common language used in dentistry. Teeth are categorized into two main classes, anterior teeth which rip food apart and posterior/molar teeth which chew the food. All anterior teeth exhibit four types of surfaces and one edge on their crowns (mesial, distal, lingual and buccal), while posterior teeth have fives surfaces on their crowns (mesial, distal, lingual, buccal and occlusal). Proximal surface 
refer to any surface between two teeth, thus it can be either mesial or distal according whether it is towards or away from the midline.

Figure 2.5. Teeth anatomy: different layers of a human model tooth are shown where the enamel and dentine layers are believed to play an important role in characterizing teeth appearance the root. The gingival line midpoint is defined to be the minima (for lower jaws) or maxima (for upper jaws) point on the gingival line formed by a single tooth. On the other hand, gingival embrasure is the respective point in the open space between the proximal surfaces of two adjacent teeth in the same dental arch................................. 24

Figure 2.6. Tooth surfaces: anterior teeth have four types of surfaces, towards and away from the midline (mesial and distal resp.), towards the lips (labial) and towards the tongue (lingual). While posteriors exhibit an additional surface for chewing (occlusal), besides the inside surface is named buccal. 25

Figure 2.7. Illustration of the proposed human jaw anatomical landmarks. The upper left corner shows a sample detected landmarks on a real image using Active Shape Model $[72]$. 26

Figure 4.1. 3D-Shape of Jaw from Statistical shape from shading with the Landmarks on one tooth for registration approach. 45

Figure 4.2. 3D-Shape of tooth from shape from shading with the Landmarks on it for registration approach. 46

Figure 5.1. Sample reconstruction result for a Lower (post-repair) jaw( second row the Image, recovered shape and groundtruth with the landmarks of the Input Image for certain that the recovered shape take the same shape of the groundtruth). Third row show from another scene for the occlusal surface. 51

Figure 5.2. Sample reconstruction result for a Upper (post-repair) jaw( second row the Image, recovered shape and groundtruth with the landmarks of the Input Image for certain that the recovered shape take the same shape of the groundtruth). Third row show from another scene for the occlusal surface. 52

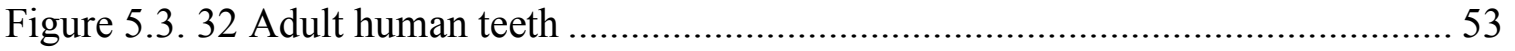

Figure 5.4. Sample CT scans for various human tooth types. (a) Maxillary deciduous teeth. (b) Maxillary molars. (c) Mandibular molars. (d) Mandibular third molars........... 54

Figure 5.5. (a) Images for different types of human teeth, captured by the intra-oral camera. (b) Groundtruth occlusal surface generated from CT scanning. Surface

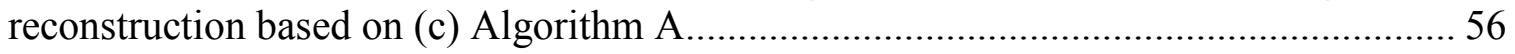

Figure 5.6. The Input Image and the difference between the SSFS and SFS approach in $3 \mathrm{D}$ construction for a jaw and comparing them with groundtruth (CT scanning)...... 57

Figure 5.7. Input Image with the Landmarks (Landmarks illustrate in red color points). 58 
Figure 5.8.Show the 3D construction for the Input Image (Figure 5.7) jaw with a different

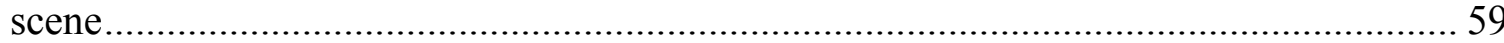

Figure 5.9. 3D- shape for the jaw with the albedo recovered ....................................... 59

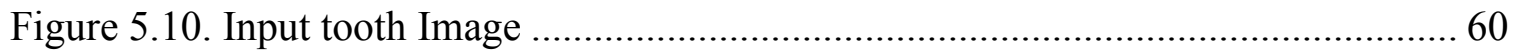

Figure 5.11. 3D reconstructions with different scenes for the tooth Image from shape

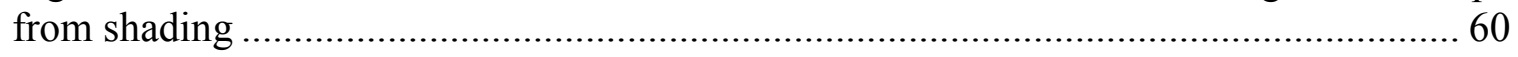

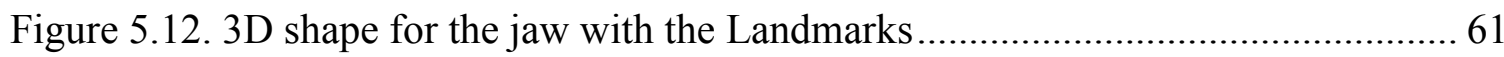

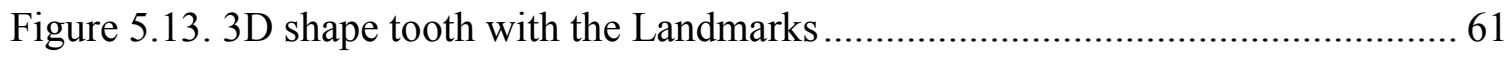

Figure 5.14. 3D shape with different scenes obtained from the Integration approach between the statistical shape from shading (SSFS) and shape from shading (SFS)........ 63

Figure 5.15. Graphic User Interface Approach for Jaw Construction Shape ................... 64 


\section{CHAPTER 1 INTRODUCTION}

Shape from shading (SFS) is a problem that has been studied for about four decades in the vision literature. Stated succinctly, the problem is to recover surface orientation from local variations in measured brightness. There is strong psychophysical evidence for its role in surface perception and recognition. Since the problem is an ill-posed one, a number of additional, simplifying model assumptions have been imposed in order to render it tractable.

The investigation of the SFS problem was pioneered by Horn [23]. He formulated the problem by a nonlinear first order partial differential Equation (PDE) called the image irradiance equation. This equation models the relationship between the shape of an object and its image brightness under known illumination conditions. His orthographic camera model, distant single point light source, and his Lambertian surface assumption became characteristic for numerous early SFS algorithms; see e.g. [44] for a survey.

In this work, will be proposed a novel strategy to improve the surface recovery results of SFS. We incorporate 3D shape priors in the SFS formulation. Since the target application is the human tooth reconstruction from intra-oral images, such information is statistically extracted from training 3D tooth models. This can serve in several aspects, e.g., to improve reconstruction accuracy, solve problems caused by occlusion (e.g., because of the tongue), specularity and albedo changes, and/or make up for the lack of sufficient, detailed view of a tooth. 
Furthermore, will be introduced a more realistic formulation of SFS that better considers all the components of the problem under concern, namely: the camera, the light source, and the surface reflectance. Since the image acquisition setup consists of a small wireless intraoral camera with a built-in bright light source, the camera is modelled by perspective projection, which is more practical than the common SFS assumption of orthographic projection. The light source is assumed to be located at the optical center of the camera. Under this near-illumination imaging, by taking into account the attenuation of illumination due to the distance between the light source and the surface, which helps to deal with the concave/convex ambiguity in SFS [33, 35].

Shape reconstruction from a single image, augmented with prior information, facilitates various studies and applications in art, design, reverse engineering and rapid prototyping. The use of prior models is rooted in the cue theory of depth perception, which states that humans learn the connection between cue and depth through previous experiences. This thesis, the statistically-constrained photometric shape analysis literature will be extended into a new domain, 'human jaw reconstruction, with new challenges such as high specular reflection and unfriendly environment for image acquisition.

The three-dimensional (3D) reconstruction of the visible part of the human jaw is becoming crucial for the treatment of malocclusion problems and several endodontic procedures. Meanwhile, since teeth are the most durable parts in the body, they are frequently used in forensic applications. Although computer tomography (CT) scanning would present $3 \mathrm{D}$ information, it is believed that there is no threshold radiation dose below which it is considered safe. As such, the aim at making it easy and feasible for doctors, dentists and researchers to obtain 3D models of a person's jaw in vivo, without 
ionizing radiation, using fewer sample points in order to reduce the cost and the intrusiveness of acquiring models of patients teeth/jaws over time. This is a challenging problem because of the 'unfriendly' environment of taking measurements inside a person's mouth.

In particular, the structure of the human jaw reveals what can be acquired in terms of prior information to enhance the SFS process and the overall accuracy of jaw modelling. For instance, the upper and the lower jaws are symmetric and are lined up according to specific anatomical features and landmarks. Thus, one of main objective of this thesis is to develop and validate an image-based approach for 3D reconstruction of the human jaw based on statistical SFS (SSFS), covering regions which the classical SFS approach does not handle and using scanned moulds and images of the oral cavity to estimate the shape of a human jaw in order to create a more accurate jaw 3D model.

From the practical point of view, the two commercial dental systems that showed the most promise during the last few years are the Itero (Cadance) and the Lava systems. Although the former is based on a red laser light source to capture multiple images while using parallel confocal microscopy to reconstruct the teeth surface, the latter uses pulsating visible blue light to capture a video sequence for the underlying jaw segment while using active wavefront sampling for surface reconstruction. Yet, these technologies have multiple user issues in the real world application. The probes are bulky and hard to handle, requiring multiple to many multiple scans to obtain full coverage of the oral cavity and the teeth. The Lava system requires the use of a visible powder to obtain good registration, and has definite depth of field issues. The Itero has an even heavier probe and can only capture one tooth at a time, requiring five views of each tooth, painstaking 
to obtain acceptable images. For both methods, blood and saliva cause inaccuracies. These systems are also laser based and very expensive. The image-based reconstruction approach is, on the other hand, on a factor of ten less expensive, allowing it to be affordable for most dental and orthodontist offices.

In practical, at the beginning of introduction was summarized about two aspects, first one is shape from shading and second one statistical shape from shading and was seen each one separately, by studying and comparing was find an adorable approach can integrate between these two aspects, Iterative Closest Point (ICP) algorithm which has a main purpose for integration between two shapes.

\subsection{Image Formation}

The image is a two-dimensional pattern of brightness. In order to use images to get information about the three-dimensional world, one needs to study the image formation process and understand how the brightness pattern is produced. This task can be accomplished by answering two main questions:

1. Where the image of some point will appear?

2. How bright the image of some surface will be?

The first question is related to the camera projection which can be either a perspective projection or an orthographic projection. The perspective projection is more general than the orthographic projection, but it requires more calculations.

The second question is related to surface reflection properties, the illumination conditions, and the surface orientation with respect to the camera and light sources. 
The following two subsections first give the mathematical expressions of perspective and orthographic projections and then describe a simple camera device to illustrate the relation between the brightness of image pixels and the reflections of their corresponding points on the surface.

\subsubsection{Geometric camera models}

The projection of a surface point of the three-dimensional scene into the twodimensional image plane can be described by a perspective projection or an orthographic projection.

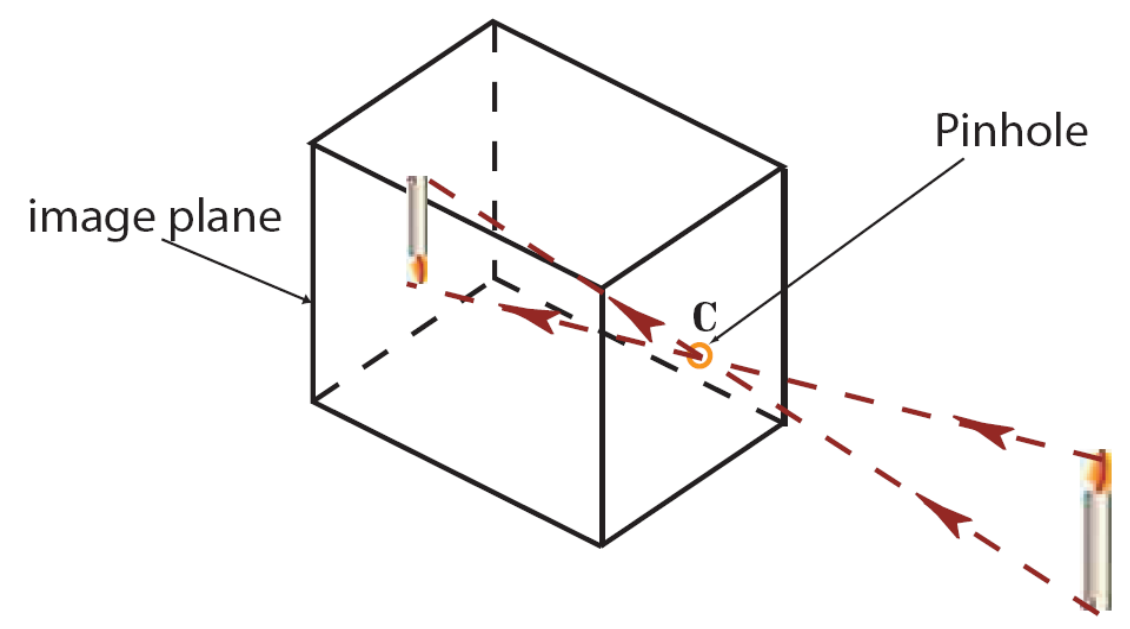

Figure 1.1. A pinhole camera produces an image that is a perspective projection of the world. 


\subsubsection{A. Perspective projection}

Figure 1.1 shows an ideal pinhole camera located at a fixed distance in front of an image plane. Assume that an enclosure is provided such that the image plane is reached only be the light coming through the pinhole. For the assumed (ideal) pinhole camera, the projection of the scene point $P$ of the $X Y Z$-space onto the image point $P$ in the $x y$-image plane is a perspective projection. The optical axis is defined to be perpendicular from the pinhole $C$ to the image plane. The distance $f$ between $C$ and the image plane is the focal length. If the coordinates system of the $X Y Z$-space is defined such that the $X Y$-plane is parallel to the image plane, and the origin is at the pinhole $C$, then the $Z$-axis lies along the optical axis.

Using the geometry shown in Figure 1.2, the equations of the perspective projection can be derived as follows:

From the two similar triangles (CÁṔ) and $(C A P)$ :

$$
\frac{f}{-Z}=\frac{i}{l}
$$

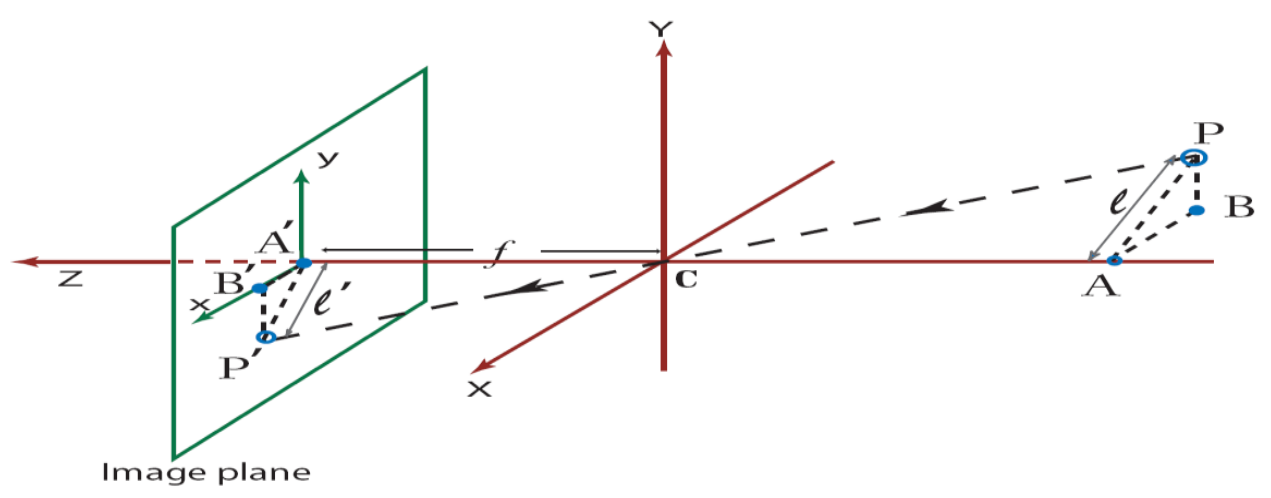

Figure 1.2. The geometry of the perspective projection. The point $\mathrm{P}=(\mathrm{X}, \mathrm{Y}, \mathrm{Z})$ is projected onto $P=(\mathrm{x}, \mathrm{y}, \mathrm{f})$ 


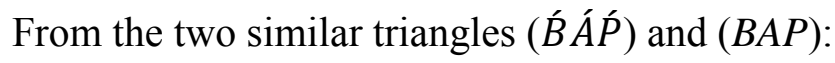

$$
\frac{x}{-X}=\frac{-y}{Y}=\frac{i}{l}
$$

From the previously mentioned two equations, the perspective projection equations are obtained:

$$
x=\frac{f X}{Z} \text { and } y=\frac{f Y}{Z}
$$

\subsubsection{B. Orthographic projection}

Suppose that the image of a p lane lying at $Z=Z o$ parallel to the image plane is formed. Then the magnification $m$ can be defined as the ratio of the distance between two points in the image to the distance between their corresponding points on the plane:

$$
m=\frac{f}{-Z_{0}}
$$

For an object located at an average distance - $Z o$ and the variations in $Z$ over its visible surface is not significant compared to -Zo (e.g., when the distance between the surface

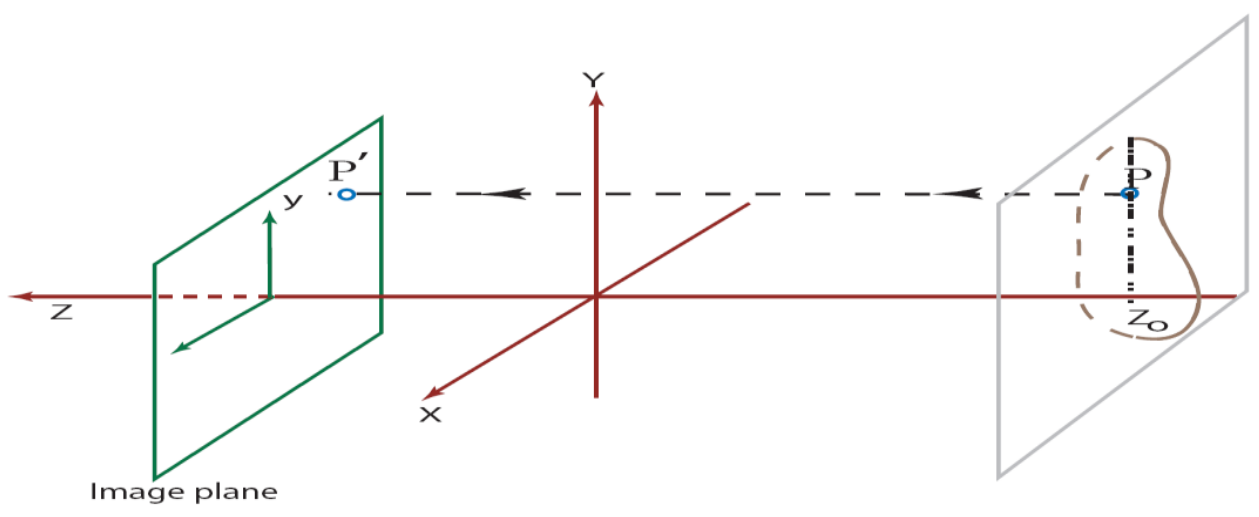

Figure 1.3. The orthographic projection. 
and the camera,-Zo, is very large relative to the variations in the object depth), then the image of this object will be magnified by $m$. For all visible points of the object, the projection equations can be simplified to:

$$
x=-m X \quad \text { and } \quad y=-m Y
$$

The scaling factor $m$ is usually set to 1 or -1 for convenience. Then the projection equations can be simplified to the orthographic projection expressed as follows:

$$
x=X \quad \text { and } \quad y=Y
$$

As shown in Figure 1.3 the orthographic projection can be modeled by rays parallel to the optical axis. The difference between the orthographic projection and the perspective projection is small when the object is located far from the camera.

\subsection{Shape From Shading VS Statistical Shape From Shading}

Statistical shape from shading (SSFS) is defined as an affordable image-based approach for reconstruction of the tooth crowns from a single occlusal image of the human jaw under arbitrary illumination based on building statistical models for the 3D shape and the 2D texture of the visible human jaw, Prior shape (full 3D), albedo (coloured) and appearance (net result of illumination and reflectance) models from the real data, which are metric in nature, are incorporated into the shape recovery framework . 
Shape from Shading (SFS) is defined as an algorithm for recovering the 3-D shape of tooth through the analysis of the brightness variation in a single image of that tooth. Two tasks have to be accomplished in solving the SFS problem. The first is to formulate an imaging model that describes the relation between the surface shape and the image brightness. This relation should consider the three components of the problem which are the camera, the light source and the surface reflectance. After establishing the imaging model, a numerical algorithm has to be developed to reconstruct the shape from the given image.

The core hypothesis of statistical shape from shading is that using prior information (full 3D) would enable obtaining a plausible and more accurate jaw model for the human jaw while covering regions that the classical SFS approach does not handle.

The output 3D-shape of tooth which obtained from shape from shading illustrated the surface detail of human tooth groove, convex and concave region.

The output 3D-shape of jaw which obtained from statistical shape from shading illustrated an accurate shape, but it lacks a detail surface of the tooth crowns.

\subsection{Thesis Organization}

The proposal is given in six chapters. Here, giving a summary of each one which includes the problem, our contribution,

\subsubsection{Chapter 1}

Gives a brief description for the thesis aspect and then explains the image formation 


\subsubsection{Chapter 2}

This chapter proposes a model-based SFS approach which allows for the construction of plausible human jaw models in vivo, without ionizing radiation, using fewer sample points in order to reduce the cost and intrusiveness of acquiring models of patients teeth/jaws over time.

\subsubsection{Chapter 3}

This chapter describes the $3 \mathrm{D}$ surface reconstruction aspect for human teeth based on a single image. A more realistic formulation of the SFS problem by considering the image formation components; the camera, the light source, and the surface reflectance is presented.

\subsubsection{Chapter 4}

This chapter proposes the Iterative Closest Point algorithm (ICP).

\subsubsection{Chapter 5}

This chapter describes and shows the experimental result from this thesis.

\subsubsection{Chapter 6}

This chapter concludes the proposed method with insights to future work expanding findings from this thesis. 


\section{CHAPTER 2 \\ 3D RECONSTRUCTION OF THE HUMAN JAW USING STATISTICAL SHAPE FROM SHADING (SSFS)}

This chapter proposes a model-based SFS approach which allows for the construction of plausible human jaw models in vivo, without ionizing radiation, using fewer sample points in order to reduce the cost and intrusiveness of acquiring models of patients teeth/jaws over time. The inherent relation between the photometric information and the underlying 3D shape is formulated as a statistical model where the effect of illumination is modeled using Spherical Harmonics (SH) and the partial least square (PLS) approach is deployed to carry out the estimation of dense 3D shapes. Moreover, shape and texture alignment is accomplished using a proposed definition of anatomical jaw landmarks which can be automatically detected. Vis-á-Vis dental applications, the results demonstrate a significant increase in accuracy in favor of the proposed approach.

In particular, this approach is able to recover geometrical details of tooth occlusal surface as well as mouth floor and ceiling as compared to SFS-based approaches.

The system is evaluated on database of 180 jaws for crown, inlay, and onlay restoration. Results show a promising performance for using the proposed approach in clinical application. 


\subsection{Introduction}

Object modeling from a single image, augmented with prior information, facilitates various studies and applications in art, design, reverse engineering, rapid prototyping and basic analysis of deformations and uncertainties. Without the use of ionizing radiation (e.g. X-ray and Computer Tomography - CT), object modeling involves constructing a 3D representation for the information conveyed in the given 2D images. This problem has been studied in the past four decades resulting in many solutions bundled under the name shape-from-X. In particular, techniques, such as SFS provide promise of imagebased 3D reconstruction when the imaging environment is somewhat precise.

To motivate the contribution of this work, dental application will be considered; 3D reconstruction of the visible part of the human jaw. Dentistry usually requires accurate 3D representation of the teeth and jaw for diagnostic and treatment purposes. For instance, orthodontic treatment involves the application, over time, of force systems to teeth for malocclusion correction. Several existing 3D systems for dental applications found in literature rely on obtaining an intermediate solid model of the jaw (cast or teeth imprints) and then capturing the 3D information from that model, e.g. [20]. There may therefore be a demand for intraoral measurement that could be fulfilled by photogrammetry, which has been applied to the measurement of many small objects, even the measurement of dental replicas. Thus photogrammetry seems to offers a reduced cost technique while avoiding the need for castings.

The argument of image-based approach for 3D reconstruction as an alternative to CTscanning. During the exposure to diagnostic imaging using x-ray (ionizing/ electromagnetic radiation), the patient body is penetrated by millions of $x$-ray photons whose 
ionization can damage the body's molecules especially DNA in chromosomes. Most DNA damage is repaired immediately, but rarely a portion of a chromosome may be permanently altered (a mutation) leading ultimately to the formation of a tumor [14]. While doses and risks for dental radiology are small, a number of epidemiological studies have provided evidence of an increased risk of brain, salivary gland [26] and thyroid tumors [22] for dental radiography. Also, pregnant mothers undergoing diagnostic or therapeutic procedures involving ionizing radiation, or who may be exposed to environmental radiation, there is a great potential for damage to the early embryo [41]. These effects are believed to have no threshold radiation dose below which they will not occur. On the other hand, CT-scanning is considered expensive and not paid by insurance companies unless disease oriented. Meanwhile, dental offices in rural areas do not have such a luxury. Thus the intent is to develop a purely image-based reconstruction mechanism as a cost-effective information tool for the dentist.

This chapter aims to make it easy and feasible for doctors, dentists, and researchers to obtain models of a person's jaw in vivo, without ionizing radiation, using fewer sample points in order to reduce the cost and intrusiveness of acquiring models of patients teeth/jaws over time. This is a challenging problem due to the "unfriendly" environment of taking measurements inside a person's mouth [42]. Further assumptions of the presence of distinct features or texture regions on the object in stereo images and the photo consistency in space carving are rarely valid in practice.

Due to the lack of surface texture, shape-from-shading (SFS) algorithms have been used to reconstruct the 3D shape of human teeth/jaw due to the significant shading cue presented in an intra-oral image, e.g. [1]. Nonetheless, in principle, SFS is an ill-posed 
problem, Prados and Faugeras [47] showed that constraining the SFS problem to a specific class of objects can improve the accuracy of the recovered shape. Thus the main objective of the presented work is to develop and validate a holistic approach for image based 3D reconstruction of the human jaw based on statistical shape from shading (SSFS), covering regions which the classical SFS approach does not handle, using scanned molds and images of the oral cavity to estimate the shape of a human jaw in order to create a more accurate jaw 3D model. The structure of human jaw reveals what can be acquired in terms of prior information to enhance the SFS process where the upper and lower jaws are symmetric and lined up according to specific anatomical features and landmarks. This approach has the potential to greatly improve plausibility of the resulting shape from shading models.

\subsection{Related Work}

There has been a substantial amount of work regarding statistical shape recovery for human face modeling and biomedical structures with distinct shapes - e.g., modeling the corpus callosum, the kidney and spinal cord; it is an active research area under shape and appearance modeling (e.g., [39, 40]). Atick et al. [5] proposed the first SSFS method where principal component analysis (PCA) was used to parameterize the set of all possible facial surfaces. Scene parameters such as pose and illumination were estimated in the process of a morphable model fitting using a stochastic gradient descent-based optimization. By considering the statistical constraint of [5] and the geometric constraint of symmetry in [45], Dovgard and Basri [17] introduced a statistical symmetric SFS method. Smith and Hancock [40] modeled surface normals within the frame- work of statistical SFS. Based on active appearance models (AAM) concept of Cootes et al. [16], 
Castelan et al. [13] developed a coupled statistical model to recover the 3D shape from intensity images with frontal light source, where the 2D shape model in [16] is replaced with a 3D shape model composed of height maps. The main advantage of the Castelan approach over the 3D morphable model framework [9] is the straightforward recovery of the 3D face shape, without undergoing a costly optimization process.

One of the main challenges that confronts SFS algorithms is dealing with arbitrary illumination. Basri and Jacobs [7] proved that images of convex Lambertian object taken under arbitrary distant illumination conditions can be approximated accurately using lowdimensional linear subspace based on spherical harmonics. This has also been validated for near illumination conditions. Since then, SH was incorporated in SFS framework to tackle the problem of illumination [4, 12, 38, 39]. Zhang and Samaras [43] primary goal was to recover the SH basis images from a single test image. Basri and Jacobs [6] relies on multiple images, i.e. photometric stereo, to infer the 3D shape. Ahmed and Farag [4] extended Castelan's coupled statistical model [13] by combining shape, appearance/albedo and $\mathrm{SH}$ in order to parameterize facial surfaces under arbitrary illumination. Rara et al. [48] further extended the work of Ahmed and Farag [4] to include 2D shape information in the model. In subsequent work, Rara et al. [38] decoupled the coupled model of [4] to obtain a separate model for shape and albedo where the classic brightness constraint in SFS is approximated using SH basis images. Castelan and Horebeek [12] and Rara [39] cast their models in a regression framework using the Partial Least Squares (PLS) method which uses a few matrix operations for shape reconstruction to provide a computationally efficient alternative to the iterative methods used in [13][38]. 


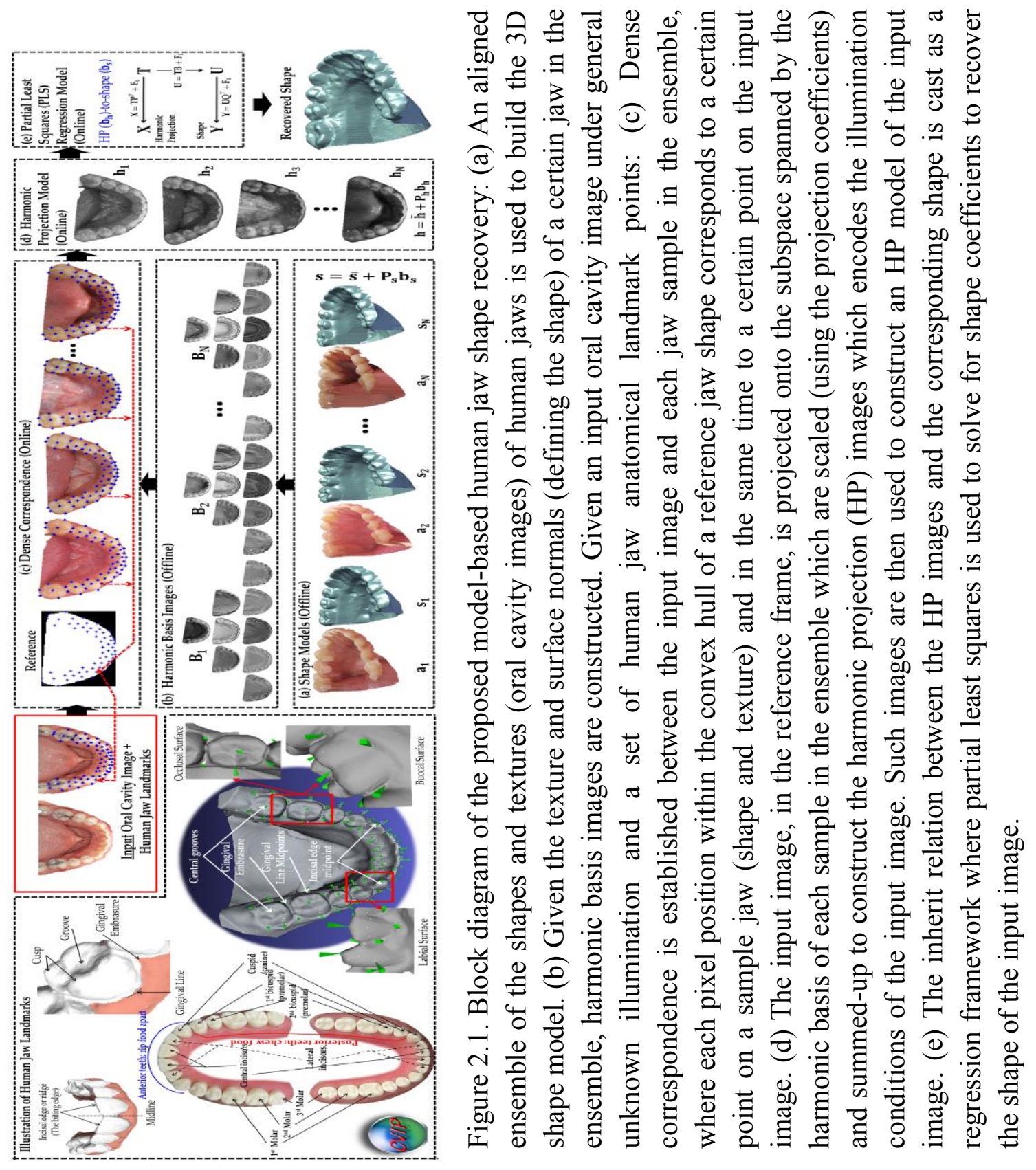




\subsection{Contributions}

This work proposes an image-based approach for reconstruction of tooth crowns which can serve as a fully automated framework for tooth restoration from a single optical image. The presented approach is based on building statistical models for 3D shape and 2D texture of human's visible jaw (clinical crowns). The process starts with annotating the jaw at known anatomical landmarks, in order to co-register the shapes and textures needed to construct the corresponding models. The key challenge is then the estimation of non-rigid transformation between an input image and the mean jaw (the origin of the object space). Such transformation is estimated using the physically motivated thin-plate splines [15] warping function using a proposed definition of anato- mical human jaw landmarks that are automatically detected. The need for hand-picked jaw features is then eliminated using an extended version of Active Shape Model (ASM) [30].

the SSFS approach will be proposed to investigate on the human jaw where face and jaw modeling carry similarities and differences. Facial images can be easily obtained and databases of various imaging conditions are already in place, along with a significant body of algorithmic development. Human faces are easy to annotate and automate the process of face cropping and feature extraction. On the other hand, the human jaw is not a friendly environment to image, as indicated before, while no databases exist to carry out a SSFS methodology.

The SSFS problem for reconstruction of the human jaw is using a series of textures and shapes (obtained from CT scans of molds) for a group of subjects. The process starts with annotating the jaw at the known anatomical landmarks, in order to co-register the shapes and textures needed to construct the corresponding models. By using spherical 
harmonics to provide the optimal basis for illumination representation and the partial least square (PLS) approach to carry out the estimation of dense 3D shapes. Key requirements for successful SSFS are the availability of a comprehensive database that describes the teeth/jaw variability per age, gender and ethnic factors. This work aims to undertake such a task and make the databases available for researchers worldwide.

\subsection{Dental Database Construction}

A major goal of this chapter is to collect resources for the jaw project in a common repository for dissemination among researchers elsewhere. At the University of Louisville Dental school there exists enormous number of molds and patient records. The first steps was taken to arrange these molds into subjects categorized with respect to gender, age and ethnicity (see Table 2.1). Using the Cone beam CT scanner of Dr. Allan Farman we scanned these molds and using the image processing tools created a mesh per mold as shown in Figure 2.2 and Figure 2.3. The data we used to generate the SSFS results were obtained from this data. The data was annotated (images and corresponding 3D mesh from the molds) and will make the data available to colleagues elsewhere. 
Table 2.1. Database construction of the human Jaw: subjects categorized with respect to gender age and ethnicity

\begin{tabular}{|c|c|c|c|c|c|c|c|}
\hline & \multirow[t]{2}{*}{ \#Teeth } & \multirow[t]{2}{*}{ Age } & \multicolumn{2}{|c|}{ Gender } & \multicolumn{2}{|c|}{ Race } & \multirow{2}{*}{$\begin{array}{l}\text { \# of } \\
\text { Jaws }\end{array}$} \\
\hline & & & Males & Females & Black & White & \\
\hline Upper & 12 & $16-46$ & 6 & 7 & 3 & 10 & \\
\hline Initial & 14 & $15-46$ & 15 & 15 & 9 & 21 & 83 \\
\hline Upper & 12 & $16-21$ & 8 & 7 & 6 & 9 & \\
\hline Final & 14 & $41-46$ & 16 & 9 & 8 & 17 & \\
\hline Lower & 12 & $11-19$ & 11 & 9 & 7 & 13 & \\
\hline Initial & 14 & $15-46$ & 17 & 18 & 13 & 22 & 97 \\
\hline Lower & 12 & $14-19$ & 5 & 6 & 2 & 9 & \\
\hline
\end{tabular}

\subsection{Anatomical Jaw Landmarks - Proposed Definition}

\subsubsection{Dental Nomenclature}

Human teeth can be classified in two groups [28]; anterior teeth which rip food apart and posterior/molar teeth which help chewing food, see Figure 2.4 for illustration. A tooth consists of several layers [46] (see Figure 2.5), however it has been believed that the enamel and dentine layers have a major impact on the visual appearance of teeth [36]; contributions from layers such as pulp may be neglected [28].

All anterior teeth have four types of surfaces: (1) Mesial - the surface toward the midline, (2) Distal - the surface away from the midline, (3) Labial - the outside surface towards the lips and (4) Lingual - the inside surface toward the tongue. Posteriors exhibit 
mesial, distal and lingual surfaces in addition to Buccal surface which is outside toward the cheek and Occlusal surface which is the chewing surface. See Figure 2.6 for illustration.

\subsubsection{Landmarks Definition}

This work mainly focuses on the reconstruction of the clinical crowns which are defined to be the portion of the teeth that is visible in the human mouth. As such, the jaw's anatomical landmarks are limited to such a space according to their location, i.e. on the tooth surface or on the interface between the tooth and the gum. Typically a landmark represents a distinguishable point which is present in most of the images under consideration, for example, the location of central grooves of each tooth. Figure 2.7 illustrates the location of 62 landmark points for a jaw containing twelve-teeth. 


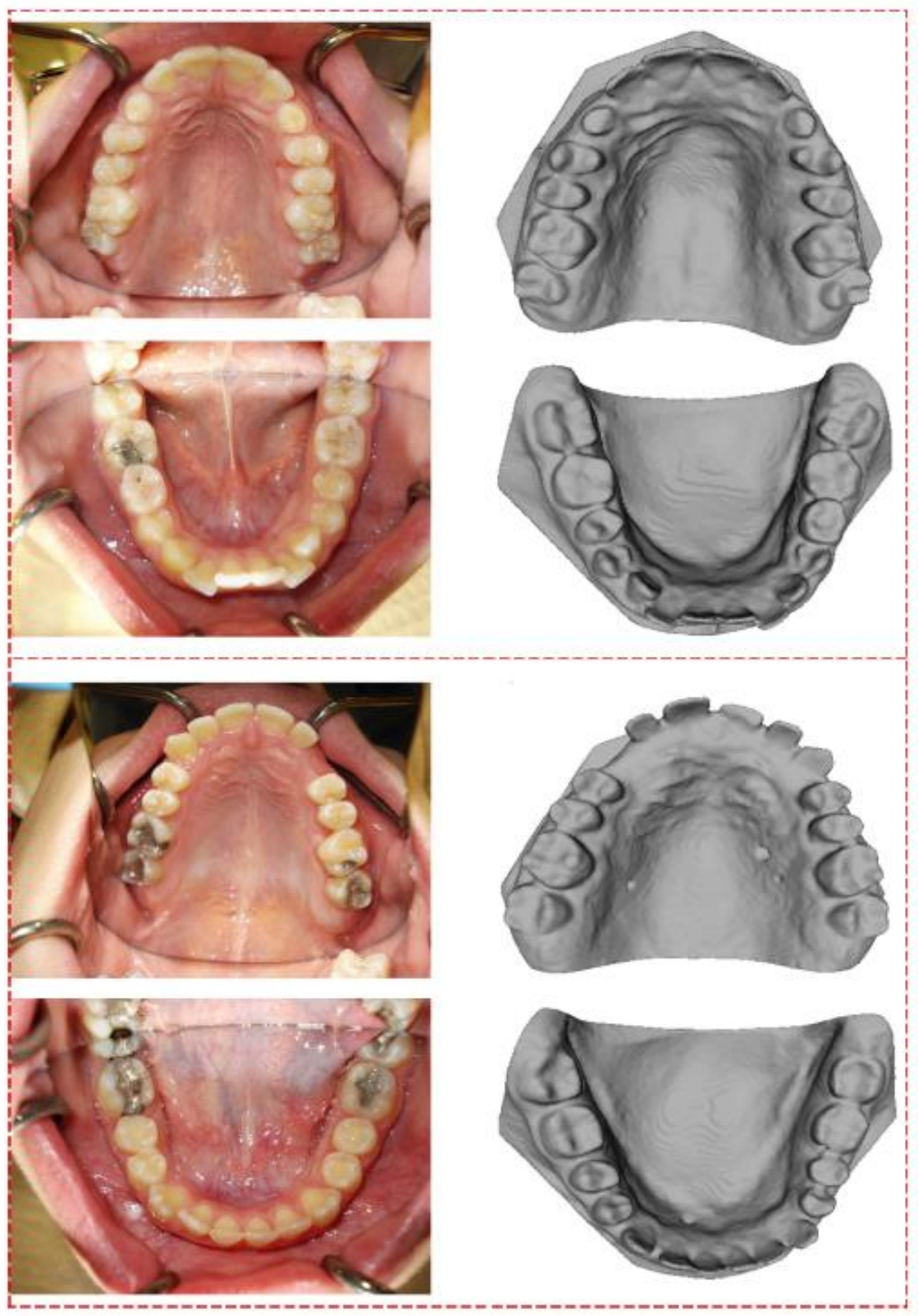

Figure 2.2. Sample of the human jaw (pre-repair) lower and upper jaws: first column shows the 2D images, 3D scans using cone beam CT machine shows in the second column. 


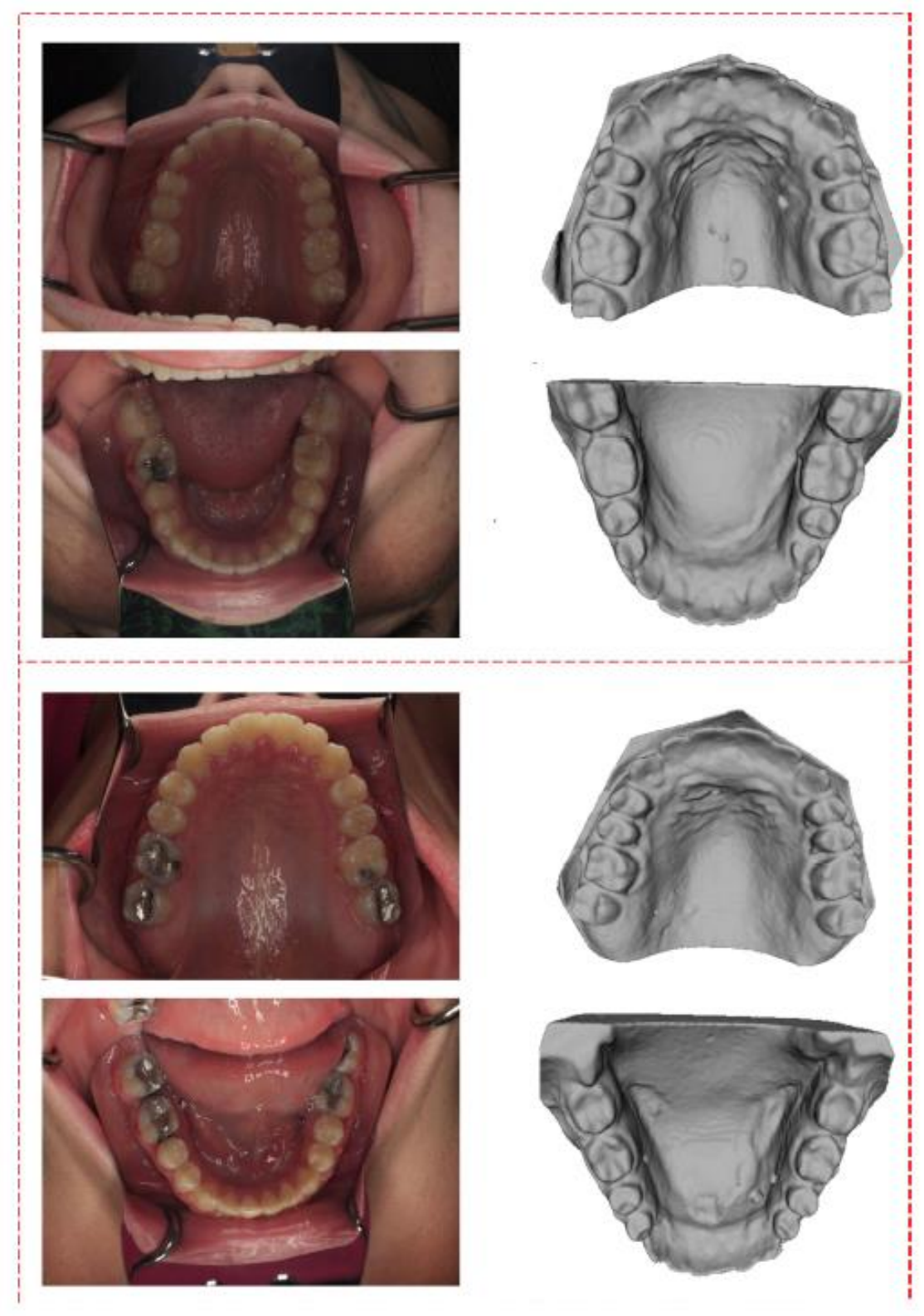

Figure 2.3. Sample of the human jaw (post-repair ) lower and upper jaws: first column shows the 2D images, 3D scans using cone beam CT machine shows in the second column. 


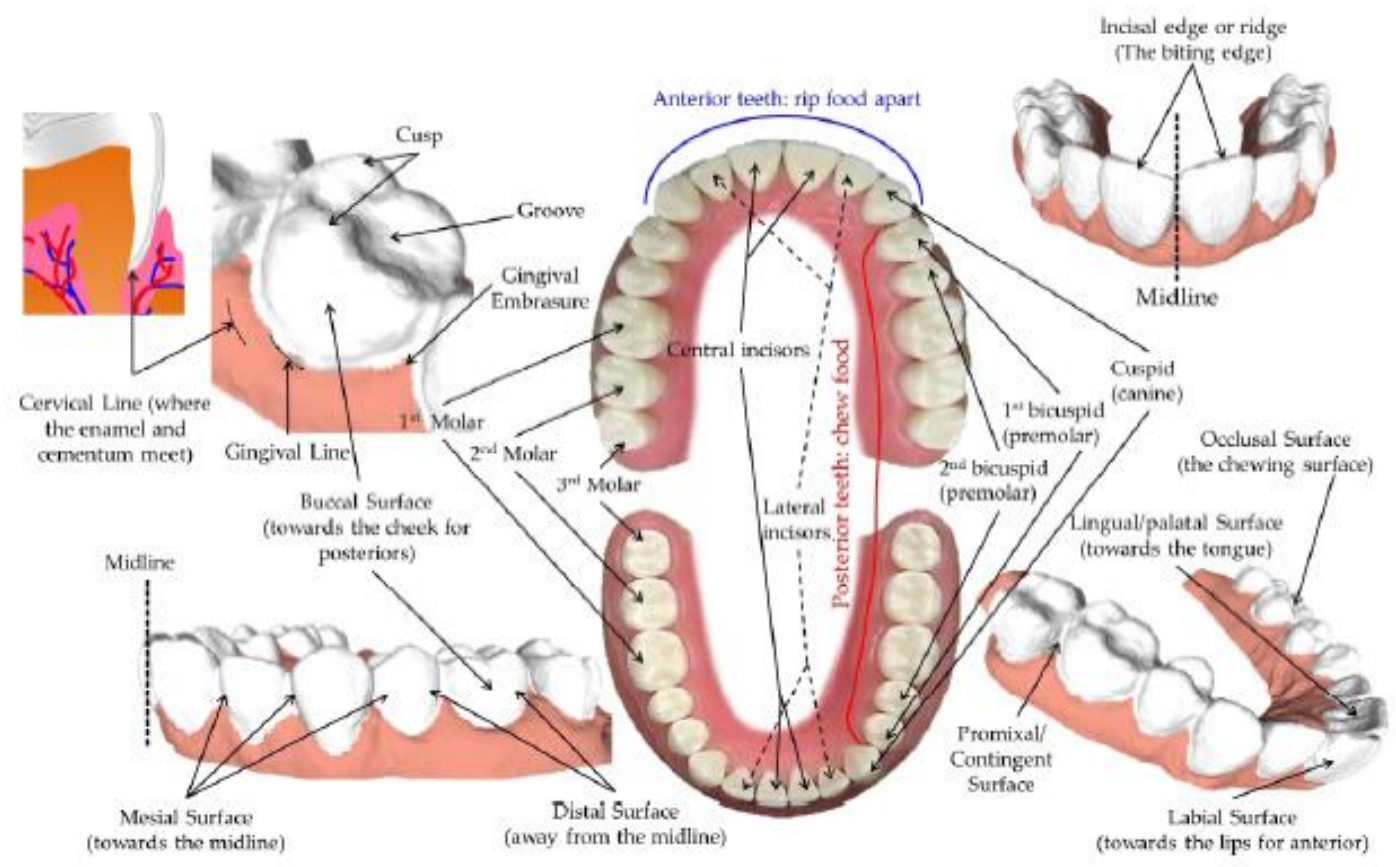

Figure 2.4. Dental nomenclature, common language used in dentistry. Teeth are categorized into two main classes, anterior teeth which rip food apart and posterior/molar teeth which chew the food. All anterior teeth exhibit four types of surfaces and one edge on their crowns (mesial, distal, lingual and buccal), while posterior teeth have fives surfaces on their crowns (mesial, distal, lingual, buccal and occlusal). Proximal surface refer to any surface between two teeth, thus it can be either mesial or distal according whether it is towards or away from the midline.

\subsubsection{A. Teeth Landmarks}

In case of posterior teeth (i.e. cuspids, premolars and molars) which are responsible for chewing food, the coalescence of the crown lobes are of interest. In particular, a central pit or groove can be considered as a landmark which is the deepest portion of a tooth fossa. While an anterior tooth (i.e. incisors) whose job is to rip food apart is identifiable by a convex elevation of the crown surface which forms the biting edge. Hence the midpoint of the incisal edge or ridge is considered as a landmark for an anterior tooth. 


\subsubsection{B. Teeth-Gum Landmarks}

The fibrous tissue covering the alveolar bone and surrounds the necks of the teeth, i.e.

The gum forms what is termed as gingival line. This line marks the level of termination of the non-attached soft tissue surrounding the tooth. It separates the clinical crown and

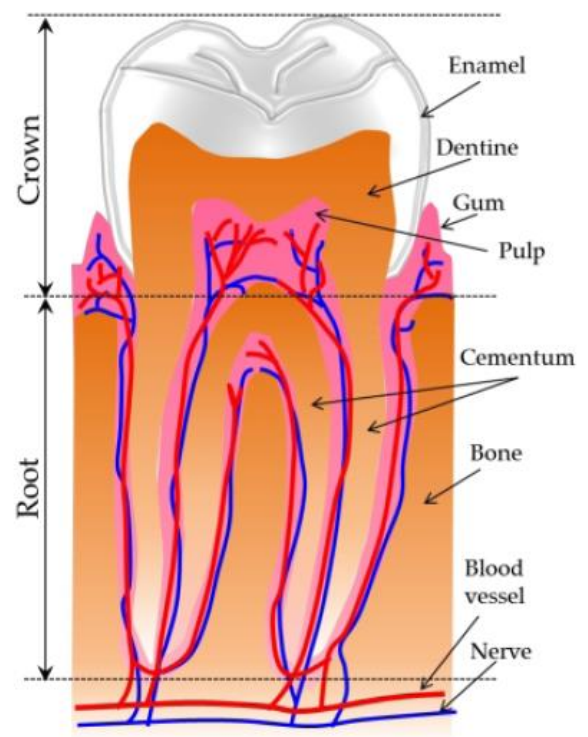

Figure 2.5. Teeth anatomy: different layers of a human model tooth are shown where the enamel and dentine layers are believed to play an important role in characterizing teeth appearance.

the root. The gingival line midpoint is defined to be the minima (for lower jaws) or maxima (for upper jaws) point on the gingival line formed by a single tooth. On the other hand, gingival embrasure is the respective point in the open space between the proximal surfaces of two adjacent teeth in the same dental arch. 


\subsubsection{Landmark Localization in Optical Images}

In the online stage of the proposed approach, a single image of the visible crowns is given from which the defined landmarks should be identified. This guides the alignment of the input image to the prior model, e.g. [8]. Hence, it is essential to automate the detection of such landmarks. In the training set, one manually annotates an ensemble of human jaws surfaces (based on CT-scanning of molds) in order to construct a sparse version of the jaw shape. These landmarks serve as a correspondence operator between individual training samples where the generalized Procrustes analysis [21] is used to

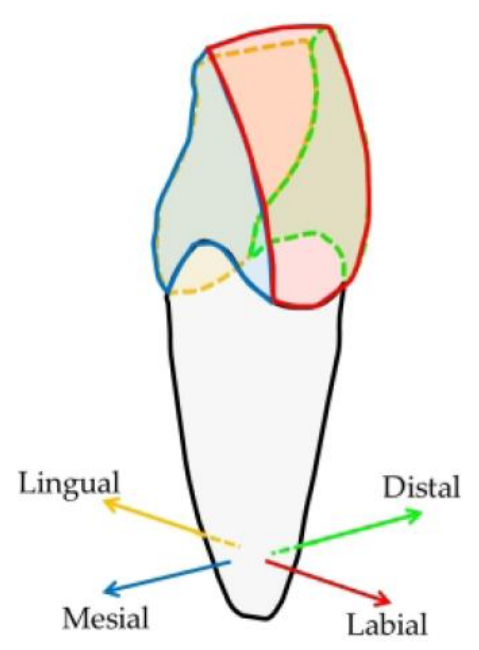

Anterior Tooth Surfaces

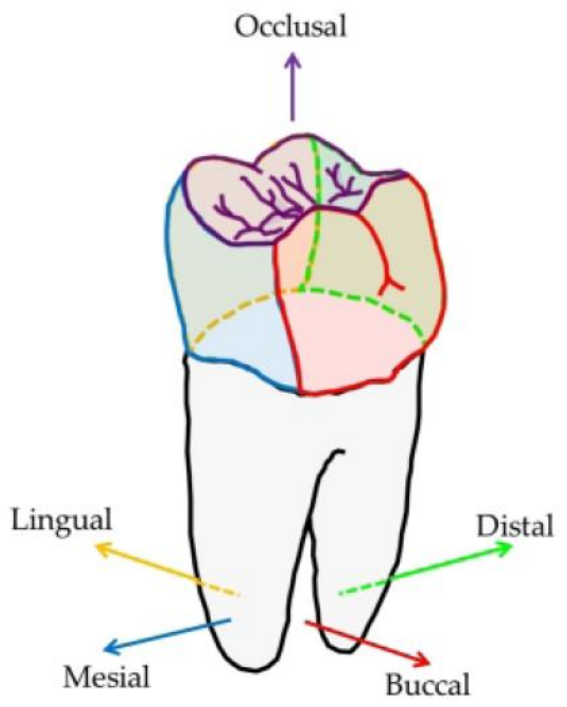

Posterior Tooth Surfaces

Figure 2.6. Tooth surfaces: anterior teeth have four types of surfaces, towards and away from the midline (mesial and distal resp.), towards the lips (labial) and towards the tongue (lingual). While posteriors exhibit an additional surface for chewing (occlusal), besides the inside surface is named buccal. 


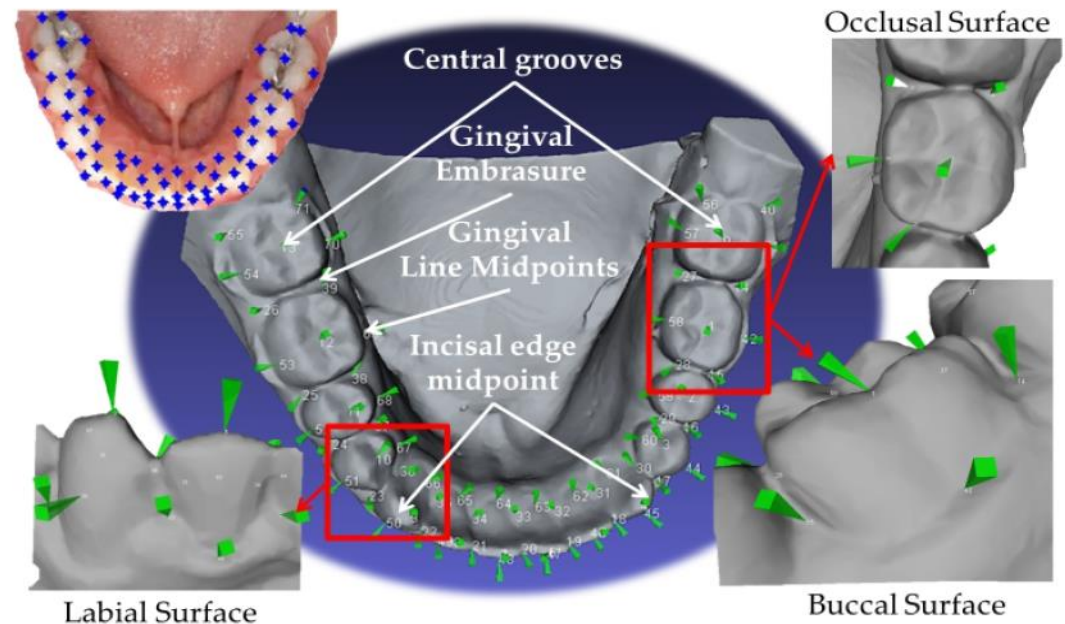

Figure 2.7. Illustration of the proposed human jaw anatomical landmarks. The upper left corner shows a sample detected landmarks on a real image using Active Shape Model [72].

filter out translation, scale and rotation. An extended version of ASM [30] is deployed to search for the landmarks in the given image. The ASM repeats the following two steps until convergence: (i) suggest a tentative shape by adjusting the locations of shape points by template matching of the image texture around each point (ii) conform the tentative shape to a global shape model. The individual template matches are unreliable and the shape model pools the results of the weak template matchers to form a stronger overall classifier. The entire search is repeated at each level in an image pyramid, from coarse to fine resolution. The initialization of the mean shape onto the given image is accomplished by segmenting the teeth region based on fitting a Gaussian mixture to the image intensity with two dominant classes; jaw and background. 


\subsection{Illumination-Invariant Statistical Shape from Shading}

When the light source and the viewer are far from the object, the image intensity $I$ at a pixel $\mathrm{x}$ can be obtained from the image irradiance of the corresponding surface point, which is defined as the surface radiance being modulated by the surface texture $a(\mathrm{x})$,

i.e. $I(\mathrm{x})=a(\mathrm{x}) \Re(\vec{n}(\mathrm{x}))$. The classical brightness constraint in SFS measures the total brightness of the reconstructed image compared to the input image, it can be defined as;

$$
\epsilon=\iint\left(I(x)-a(x) \Re(\vec{n}(x))^{2} d x\right.
$$

Where $a(:)$ is the surface texture at point $\mathrm{x}$ while $\mathfrak{R}(:)$ is the radiance of the surface patch with unit normal $\vec{n}$ (x), also known as surface reflectance function.

The brightness constraint in equation (2.1) can be rewritten in the discrete domain as a linear combination of harmonic basis images resulted from the 2nd order SH approximation to the reflectance function [7]. Thus the image intensity $I$ can be expressed as;

$$
I(\mathrm{x})=\sum_{i=0}^{n-1} a i b i(x)
$$

Where $b i(x)=f i(a(\mathrm{x}), \mathrm{n}(\mathrm{x}))$ are the harmonic basis images which are functions of surface texture $a(\mathrm{x})$ and surface normals $\vec{n}$ (x) at pixel x (refer to [7] for their definition). The coefficient $a_{i}$ denotes the ith coefficient in the illumination spectrum being modulated by the Lambertian kernel spectrum.

In matrix notation, let $I \in \mathbb{R}^{d * 1}$ be an image vector with $d$ pixels, $\boldsymbol{B}=\left[b_{0}, b_{1}, \ldots \ldots b_{n-1}(x)\right] \in \mathbb{R}^{d * n}$ be the matrix of harmonic basis images as its columns, where $\mathrm{n}$ is the number of basis images, typically $n=9$, and $a \in \mathbb{R}^{n * 1}$ vector of SH coefficients. Hence the discrete version of the brightness constraint becomes, 


$$
\epsilon=\sum_{x}^{n}(I(x)-B(x) a)^{2}=\|I-B a\|
$$

Representing the surface reflectance function in terms of $\mathrm{SH}$ allow us to infer the illumination of a given image; given an input image $I$, the harmonic basis images $\boldsymbol{B}$ of a $3 \mathrm{D}$ object (a human jaw in particular), defined by it shape $\mathrm{s}=\left[n\left(x_{0}\right), \ldots \ldots, n\left(x_{n-1}\right)\right]^{T}$ and texture $\mathrm{a}=\left[a\left(x_{0}\right), \ldots \ldots, a\left(x_{d-1}\right)\right]^{T}$, are obtained to deduce the coefficients $\mathrm{a}$ that best matches the input image. This results in an over-determined linear system of equations $I=$ Ba which can be solved for â using singular value decomposition (SVD).

If the input image and the basis images used to compute the coefficients â belong to the same object, the input image was constructed by these coefficients, i.e. $h=\boldsymbol{B} \hat{\mathrm{a}}=I$, where $h$ denotes what we call harmonics projection (HP) image. However in the general case, the basis images $\boldsymbol{B}$ would belong to an object which is different from the one in the input image $I$, nonetheless they belong to the same object class e.g. different realizations of a human jaw. Thus the reconstructed image $h$ provide a mean of encoding the illumination of the input image while maintaining the identity of the object whose basis images are used in the reconstruction process.

While Equation (2.1) can be solved in an iterative manner to infer the underlying shape as in [38], the inherent relation between the HP images $h$ and the corresponding shape $\mathbf{s}$ can be cast into a regression framework resulting into the HP-to-shape model. In this case, the shape is solved for using a series of matrix operations guaranteeing faster shape recovery when compared to its iterative counterpart. This was proven to yield comparable results in terms of reconstruction accuracy [39]. 
Dimensionality reduction is performed using PCA to construct the 3D shape model (offline step) and HP model (online step) where the coefficients are used to build the regression model rather than the original shape and HP instances. In particular, the 3D shape model can be constructed by performing PCA on a set of aligned samples of 3D shapes, the resulting shape model is $\boldsymbol{s}=\overline{\boldsymbol{s}}+\boldsymbol{P}_{\boldsymbol{s}} \boldsymbol{b}_{\boldsymbol{s}}$ where $\overline{\boldsymbol{s}}$ is the mean shape, $\boldsymbol{P}_{\boldsymbol{s}}$ are the shape eigenvectors and $\boldsymbol{b}_{\boldsymbol{s}}$ is the set of shape coefficients. On the other hand, the HP model is trained online which incorporate the illumination conditions of the input image; given an image $I$ and the basis images $\boldsymbol{B}_{\boldsymbol{k}}$ of object instance k, the HP image $\boldsymbol{h}_{\boldsymbol{k}}$ is obtained, where $\boldsymbol{h}_{\boldsymbol{k}}=\boldsymbol{B}_{\boldsymbol{k}} \hat{\mathrm{a}}_{\boldsymbol{k}}$ with $\hat{\mathrm{a}}_{\boldsymbol{k}}$ obtained by solving the linear system of equations $I=\boldsymbol{B}_{\boldsymbol{k}} \mathrm{a}_{\boldsymbol{k}}$. After reconstructing the projection images of all the instances in the jaw database, we can model the HP images using PCA as $h=\overline{\boldsymbol{h}}+\boldsymbol{P}_{\boldsymbol{h}} \boldsymbol{b}_{\boldsymbol{h}}$ where $\overline{\boldsymbol{h}}$ is the mean HP image, $\boldsymbol{P}_{\boldsymbol{h}}$ are the HP images eigenvectors and $\boldsymbol{b}_{\boldsymbol{h}}$ is the set of HP coefficients. Thus, instead of using the high dimensional vectors $\boldsymbol{b}_{\boldsymbol{s} \boldsymbol{k}}$ and $\boldsymbol{h}_{\boldsymbol{k}}$ into the regression, they are replaced by their respective coefficients $\boldsymbol{b}_{\boldsymbol{s} \boldsymbol{k}}$ and $\boldsymbol{b}_{\boldsymbol{h} \boldsymbol{k}}$, where the HP coefficients are considered the independent variable while the shape coefficients are the dependent variables. Following [39], we use partial least squares regression (PLS) instead of the classical least squares to avoid random noise which might exist in the dependent and independent variables. Figure 2.1 shows a block diagram of the offline/online processes for the proposed shape recovery approach.

Moreover as we saw recently that we modeled the HP image model by using PCA as , in the same concept we model a sparse 2D model Landmarks for annotation landmarks by using PCA as $\boldsymbol{S}_{2 D}=\overline{\boldsymbol{s}}_{2 \boldsymbol{D}}+\boldsymbol{P}_{\boldsymbol{S 2 D}} \boldsymbol{b}_{\boldsymbol{s} 2 \boldsymbol{D}}$. We use the partial least squares regression(PLS) for “ $\left(b_{s 2 D}\right)$ to Shape $\left(b_{s}\right)$ ". 


\subsection{Summary}

This chapter presented an affordable, flexible, automatic dental tool for the reconstruction of the clinically visible part of the human jaw. It was based on a single captured optical image and a statistical shape recovery approach which makes use of a small number of measured points to construct a plausible $3 \mathrm{D}$ model through a learned correspondence based on a measured human jaw dataset. The surface reflectance function is expressed in terms of spherical harmonics to provide the optimal basis based on training data for illumination representation. The brightness constraint was then cast as a Partial Least Squares (PLS) regression problem, which allows for the rapid computation of the solution. The PLS algorithm is composed of a sequence of matrix operations; the approach in this work can recover 3D shapes much faster than its iterative counterpart, without compromising the integrity of the results. The results demonstrated the effect of adding statistical prior as well as illumination modeling on the accuracy of the recovered shape. 


\section{CHAPTER 3 \\ 3D RECONSTRUCTION OF THE HUMAN TEETH USING SHAPE FROM SHADING (SSFS)}

Image formation involves understanding sensor characteristics and object reflectance. In dentistry, an accurate 3D representation of the human jaw may be used for diagnostic and treatment purposes. Photogrammetry can offer a flexible, cost effective solution for accurate 3D representation of the human teeth, which can be used for diagnostic and treatment purposes. Nonetheless there are several challenges, such as the non-friendly image acquisition environment inside the human mouth, problems with lighting and errors due to the data acquisition sensors. In this chapter, the 3D surface reconstruction aspect will be focused for human teeth based on a single image. A more realistic formulation of the SFS problem will be introduced by considering the image formation components; the camera, the light source, and the surface reflectance. We propose a nonLambertian SFS algorithm under perspective projection which benefits from camera calibration parameters. The attenuation of illumination due to near field imaging will be taken into account. The surface reflectance is modeled using Oren-Nayar-Wolff model which accounts for the retro-reflection case. 


\subsection{Introduction}

Modern dentistry requires the accurate $3 \mathrm{D}$ representation of the teeth and jaw for diagnostic and treatment purposes. For instance, orthodontic treatment involves the application, over time, of force systems to teeth for malocclusion correction. Oral and maxillofacial radiology can provide the dentist with abundant 3D information about the jaw. Several existing 3D systems for dental applications found in literature rely on obtaining an intermediate solid model of the jaw (cast or teeth imprints) and then capturing the $3 \mathrm{D}$ information from that model. Due to the lack of surface texture, SFS algorithms have been used to obtain such 3D tooth reconstructions due to the significant shading cue presented in a tooth image, e.g. $[1,11]$.

Photogrammetry seems to offer a flexible, cost effective solution while avoiding the need for castings. Nonetheless, intra-oral photogrammetric measurement is inherently difficult due to non-friendly image acquisition environments with lighting problems, specularity effects due to saliva, inevitable subject motion and errors due to the data acquisition sensors [31, 42]. Hence the common assumptions of the image formation process for typical shape reconstruction algorithms are hardly valid, e.g. Lambertian reflectance and distant light source.

Starting from the pioneering work of Horn [25], shape recovery from a single image usually involves two steps; deriving an image irradiance equation under a certain set of assumptions related to the image formation process and designing a numerical scheme to solve such an equation for the underlying shape. Most of the SFS approaches (e.g. see [18]), however, focus on the computational part of the SFS problem, i.e. the numerical solution. As a result, the imaging model in most conventional SFS algorithms has been 
simplified under three simple, but restrictive assumptions: (1) the camera performs an orthographic projection of the scene, (2) the surface has a Lambertian reflectance and (3) the light source is a single point source at infinity. Unfortunately, such assumptions are no longer held in the case of intra-oral imaging environment for human teeth.

This chapter introduces a more realistic formulation of SFS by considering all the components of the problem, namely: the camera, the light source, and the surface reflectance. For the camera and the light source use the same modeling as in [34]. The camera will be modeled by perspective projection with the camera parameters being known from an offline calibration process and incorporated in the SFS formulation. The light source is assumed to be located at the optical center of the camera, i.e. retroreflection situation where the object is illuminated in the viewing direction. Under this near-field imaging, the attenuation of illumination was taken into account due to the distance between the light source and the surface; hence our method can deal with concave/convex ambiguity. Accounting for departures from Lambertian reflectance due to surface roughness, we use a modified Oren-Nayar-Wolff model [27] where surface roughness is physically measured using optical surface profiler. The Oren-Nayar model [32] modulates the Lamberts cosine law by a term that depends on the squared sine of the incidence angle, resulting in apparent brightening at surface patches which move away from the light source; assumed to be in self shadow. Wolff, on the other hand, has a physically deeper model for diffuse reflectance from shiny but slightly rough surfaces. The model uses an angle dependent Fresnel term to account for the refractive attenuation of incident light at the surface-air boundary [37]. This Fresnel term modifies the 
Lambertian cosine model in a multiplicative way. The effect is to depress the surface radiance for near-normal incidence.

The numerical solution, classified as Minimization-based [18], is based on the Taylor series expansion of the image irradiance equation followed by the application of the Jacobi iterative method. On the other hand, the image irradiance equation can be formulated as a partial differential equation (PDE) to solve for surface gradients, where the theory of viscosity solutions for Hamilton-Jacobi type equations provide a good framework of SFS algorithms [18]. However explicit PDE formulation of the SFS problem imposes regularity of the image irradiance function which is assumed to be continuous [18]. Nonetheless, human teeth do not fit such an assumption, due to the geometrical structure of the occlusal surface in particular, which forms attached and cast shadows in the captured image causing image discontinuities.

Carter et al. [11] evaluated three SFS models for artificial tooth surface reconstruction based on the work of Ahmed and Farag [2]. They concluded that, based on the quantitative error analysis, a perspective camera projection with an Oren-Nayar reflectance model has been proved to be the most ideal SFS formulation for extracting tooth crown surface from a single image. Nonetheless, their work did not incorporate the available camera parameters from their acquisition setup nor the object physical characteristics into the SFS-PDE formulation. The proposed SFS approach is compared with a non-Lambertian PDE-based approach $[3,11]$ via quantitative error metric derived from groundtruth teeth surfaces obtained from a CT-scanner. Vis-á-Vis dental applications, the results demonstrate a significant increase in accuracy in favor of the 
proposed approach. In particular, this approach is able to recover geometric details of the tooth's occlusal surface as compared to PDE-based approaches.

\subsection{Image Irradiance Equation}

According to the microscopic view of occlusal surface height variations, tooth surface reflectance can be modeled by micro-facet reflectance models where the Oren-NayarWolff model is well-suited for the retro-reflection case [37]. When the object is illuminated in the viewing direction, taking into account the illumination attenuation term $\left(1 / r^{2}\right)$, the expression of the image irradiance E using Oren-Nayar-Wolff model can be simplified to [27]:

$$
\begin{aligned}
& \mathrm{E}(\mathrm{x})=\frac{A(1-F(\theta, n))^{2} \cos \theta+B \sin ^{2} \theta}{r^{2}} \\
& \text { s.t. } A=1-0.5 \frac{\sigma^{2}}{\sigma^{2}+0.33}, B=0.45 \frac{\sigma^{2}}{\sigma^{2}+0.09} .
\end{aligned}
$$

Where $r$ is the distance to the light source, the parameter $\sigma$ denotes the standard deviation of the Gaussian distribution which is used as a measure of the surface roughness, $\theta$ is the inclination angle of the viewer/source and $F$ refer to the Fresnel reflection function [27] with refractive index of $n$. 


\subsection{Parametric SFS for Non-Lambertian Surfaces}

A surface point in the 3D space can be related to its corresponding position in the image plane through the camera intrinsic (solved for once) and extrinsic (updated while the camera is in motion) parameters. The relation between a 3D point $\mathrm{X}=(X, Y, Z)^{T}$ and the corresponding point in the image coordinates $\mathrm{x}=(x, y)^{T}$ is written as $\mathrm{s}(\mathrm{x}) \vec{x}=\mathrm{PX}+\mathrm{b}$, where $\mathrm{s}$ is a scalar parameterized by the image pixel coordinates, $\mathrm{P}$ is a $3 \times 3$ camera matrix, $\mathrm{b}$ is a $3 \times 1$ translation vector and $\vec{x}=\left[x^{T} 1\right]^{T}$ is the extended vector defined in homogeneous coordinates. Therefore the point in $3 \mathrm{D}$ coordinates can be written as a function of the corresponding point in the image plane as,

$$
X=P^{-1}[s(x) \vec{x}-b]=g(x(s))
$$

Equation (3.2) represents the line in 3D passing through the optical center and the projected point $\mathrm{x}$ in the image plane. Thus solving for the scalar $\mathrm{s}(\mathrm{x}) \forall x \in \mathbb{R}^{2}$, using the available shading cues in the image domain $\mathcal{D} \in \mathbb{R}^{2}$, accounts for defining a unique 3D point $\mathrm{X}$ on the object's surface. The inherent relationship between the scale factor $\mathrm{s}(\mathrm{x})$ and the surface normal vector $\vec{n}(\mathrm{x})$ can be expressed in terms of surface gradients $(\mathrm{p}(\mathrm{x}), \mathrm{q}(\mathrm{x}))$ in the gradient space [24], where $\mathrm{p}(\mathrm{x})=\frac{\partial g(s(x))}{\partial x}=\mathrm{p}(\mathrm{s})$ and $\mathrm{q}(\mathrm{x})=\frac{\partial g(s(x))}{\partial y}=$ $\mathrm{q}(\mathrm{s})$. The image irradiance $E($.$) now becomes a function of the scalar \mathrm{s}(\mathrm{x})$ defined in equation(3.2). In the sequel, the variation formulation was presented for the problem in case of near illumination with Oren-Nayar-Wolff reflectance. 


\subsection{Summary}

This chapter focused on the surface reconstruction aspect of human teeth from a single image captured by an intra-oral camera under near-field imaging. This work has addressed several challenges related to the image formation process including near illumination, perspective projection, while taking into account the deviation from the simplifying Lambertian assumption. the formulation exploits all calibration information provided by the acquisition system setup. In particular, on average, this approach reduces the error metric by $0,4434 \mathrm{~mm}$ compared to un-calibrated PDE-based SFS formulation. While the improvements are fractions of a millimeter, this is considered significant for dental-related applications such as tooth implant and surface analysis. 


\section{CHAPTER 4 \\ ITERATIVE CLOSEST POINT TECHNIQUE FOR 3D SHAPE REGISTRATION}

In this chapter, a convenient 3D shape registration technique based on the Iterative Closest Point (ICP) mapping function was presented. The method can determine the corresponding 3D shape information and obtain the matching shape structure from different shapes. Using this technique, the two sets of shape coordinate information used as the mapping parameters obtained from the relative shapes with different techniques were chosen from a developed computer user interface manually. The selected shape coordinate points were used to find out the coordinate relationship of each shape information to decide the mapping function. Furthermore, the deformed shapes and registration results can be integrated by the determined mapping function.

\subsection{Introduction}

Shape from Shading (SFS) algorithm in (chapter 3) has been used to reconstruct the 3D shape of human teeth due to the significant shading cue presented in an intra-oral image. Nonetheless, in principle, SFS is an ill-posed problem, Prados and Faugeras [47] showed that constraining the SFS problem to a specific class of objects can improve the accuracy of the recovered shape. Also the statistical shape from shading (SSFS) algorithm (chapter 2) has been used to reconstruct the 3D shape of human jaw and covering regions which the classical SFS approach does not handle, using scanned molds and images of the oral 
cavity to estimate the shape of a human jaw in order to create a more accurate jaw 3D model.

Due to the lack of surface depth from statistical shape from shading, an expected tool was developed that deformed and reconstructed shape to obtain integrated information of two approaches; Shape from shading (SFS) and statistical shape from shading (SSFS). The developed tool used a method called Iterative Closest Point (ICP) [49] mapping method. ICP algorithm function can be used to determine a deformation function according to two sets of corresponding control points on the relative shapes.

\subsection{Related work}

The original ICP algorithm has been widely applied to rigid registration, but it does not work well in non-rigid registration problems. [50] Proposed an extension of the ICP Algorithm for scaling registration. [51] Used an additional stiffness term and a landmark term for locally affine regularization, which is based on the extended ICP algorithm. Some scholars managed to solve non-rigid registration problems but they avoided using the ICP algorithm. [52] Proposed a robust approach for non-rigid registration by using mixture of Gaussian. [53] And [54] presented algorithms to register multiple unlabeled point sets to an emerging mean shape. In non-rigid registration, affine registration is an important and key problem. When affine transformation is considered to register two point sets without noise, the error of least square (LS) problem is 0, but affine transformation is not unique. For example, if two point sets are best matched with the true affine transformation, the error is 0 . However, if the affine transformation is close to 0 , that is, all points of one point set are transformed to register one point of the other point set, and then the error is also close to 0 . To solve this ill-posed problem, the usual 
way is to add regularization terms. [55] Introduced the normal and the principal curvatures in points coordinates. It is apparently time-consuming to compute the normal and the principal curvatures and search the closet points. Meanwhile, much information of point sets is needed. For instance, the interior and the exterior of objects need to be known to obtain the orientation of the normal. Moreover, some scholars managed to avoid using optimization to guarantee uniqueness of affine transformation. [56] [Reduced the general affine registration problem to that of the orthogonal case with covariance matrices]. However, its result is not quite accurate. In addition, some scholars have tried to use probabilistic point set registration methods which soft assign correspondence for registration. [57] Proposed the thin plate spline_ robust point match (TPS_RPM) algorithm which used the soft assignment of weights for non-rigid registration. Furthermore, probabilistic point matching (PPM) and coherent point drift (CPD) were proposed by [59], [59] and [60], respectively. These methods need to establish correspondence of all point sets, so their speed is much slower.

\subsection{The ICP Algorithm}

[61] Described a LS problem in which rigid transformation was considered in point set registration. Given two overlapping point sets in $\mathbb{R}^{3}$, a data shape $P \triangleq\left\{\vec{p}_{i}\right\}_{i=1}^{N_{p}}\left(N_{p} \in \mathbb{N}\right)$ and a model shape $M \triangleq\left\{\vec{m}_{i}\right\}_{i=1}^{N_{m}}\left(N_{m} \in \mathbb{N}\right)$. A rigid registration between two 3D point sets is to find rotation and translation transformations, with which $\mathrm{P}$ is in the best alignment with $\mathrm{M}$, then the formulation is given here based on LS criterion as follows: 


$$
\min _{\boldsymbol{R}^{\boldsymbol{T}}, \vec{t}, j \in\left\{1,2, \ldots, N_{m}\right\}}\left(\sum_{i=1}^{N_{p}}\left\|\left(\boldsymbol{R} \vec{p}_{i}+\vec{t}\right)-\vec{m}_{j}\right\|_{2}^{2}\right)
$$

$$
\text { s.t. } \boldsymbol{R}^{T} \boldsymbol{R}=I_{3}, \operatorname{det}(\boldsymbol{R})=1 \text {, }
$$

Where $\boldsymbol{R} \in \mathbb{R}^{3 \times 3}$ is a rotation matrix, $\vec{t} \in \mathbb{R}^{3}$ is a translation vector.

The ICP algorithm achieves rigid registration with good accuracy and fast speed mainly in two steps.

Firstly, built up correspondence between two point sets:

$$
C_{k}(i)=\underset{j \in\left\{1,2, \ldots, N_{m}\right\}}{\arg \min }\left(\left\|\left(\boldsymbol{R}_{k-1} \vec{p}_{i}+\vec{t}_{k-1}\right)-\vec{m}_{j}\right\|_{2}^{2}\right), i=1, \ldots \ldots, N_{p}
$$

Secondly, compute the new rotation and translation transformations by minimizing squared distance:

$$
\left(R_{k}, t_{k}\right)=\underset{\boldsymbol{R}^{T} \boldsymbol{R}_{I_{3}, \operatorname{det}(\boldsymbol{R})=1, \vec{t}}}{\arg \min }\left(\sum_{i=1}^{N_{p}}\left\|\boldsymbol{R} \vec{p}_{i}+\vec{t}-\vec{m}_{C_{k}(i)}\right\|_{2}^{2}\right)
$$

\subsection{Affine Registration}

In this section, to get the best affine registration between two 3D point sets, a general LS problem is proposed.

\subsubsection{Problem Statement}

Affine registration of $3 \mathrm{D}$ point sets is an arduous work. To tackle it, a general statement is described as follows. Given two point sets in $\mathbb{R}^{3}$ and assume one point set can be transformed with an affine transformation to match a subset of the other point set. To 
register between these two 3D point sets is to find an affine transformation $T$, with which $P$ is registered to be in the best alignment with $M$. Hence the affine registration between two point sets is based on the following LS criterion:

$$
\min _{T, j \in\left\{1,2, \ldots, N_{m}\right\}}\left(\sum_{i=1}^{N_{p}}\left\|T\left(\vec{p}_{i}\right)-\vec{m}_{j}\right\|_{2}^{2}\right) .
$$

To simplify this objective function, $T$ needs expressing in a more explicit form. Firstly of all, the affine transformation $\mathrm{T}$ is decomposed into an invertible matrix $\boldsymbol{A}$ and a translation vector $\vec{t}$, so Equation (4.4) is rewritten as

$$
\min _{A, \vec{t}, j \in\left\{1,2, \ldots, N_{m}\right\}}\left(\sum_{i=1}^{N_{p}}\left\|\left(\boldsymbol{A} \vec{p}_{i}+\vec{t}\right)-\vec{m}_{j}\right\|_{2}^{2}\right) .
$$

Secondly, according to singular value decomposition (SVD) method, an invertible matrix with real coefficients $\boldsymbol{A} \in G L(3, R)$ is decomposed into three special matrices: two orthogonal matrices $\boldsymbol{U}$ and $\boldsymbol{V}$, a diagonal matrix with positive entries $\boldsymbol{S}$. Namely, $\boldsymbol{A}=$ $U S V^{T}$. Because $V^{T}$ and $V$ are all orthogonal matrices, without loss of generality, by assuming $\boldsymbol{R} \triangleq V^{T}$, so $\boldsymbol{R}$ is still an orthogonal matrix. By substituting this in equation (4.5), the objective function can be formulated as

$$
\min _{\boldsymbol{U}, \boldsymbol{R}, \boldsymbol{S}, \vec{t}, j \in\left\{1,2, \ldots, N_{m}\right\}}\left(\sum_{i=1}^{N_{p}}\left\|\left(\boldsymbol{U S} \boldsymbol{R} \vec{p}_{i}+\vec{t}\right)-\vec{m}_{j}\right\|_{2}^{2}\right)
$$


As is known to all, an affine transformation is a combination of a series of basic transformations, such as translation, reflection, rotation and scale transformations. In equation (4.6), the orthogonal matrices $\boldsymbol{U}$ and $\boldsymbol{R}$ both stand for reflection and rotation transformations while $S$ represents scale transformation.

\subsubsection{The Affine ICP Algorithm}

As reflection and rotation transformations are two different transformations and reflection transformation can be given at the initial stage of the registration like the ICP algorithm, rotation transformation is to be considered. Moreover, there is a phenomenon in Equation (4.6) that points of a set may converge to a point of the other set, that is, the affine transformation is close to 0 . According to $|\operatorname{det}(A)|=|\operatorname{det}(U) \operatorname{det}(S) \operatorname{det}(R)|=$ $|\operatorname{det}(S)|$, the scale transformation is close to 0 . A method to avoid this phenomenon is to bound the scale matrix $\mathrm{S}$ in Equation (4.6). With the exposition above, the affine registration problem now becomes the following constrained optimization problem:

$$
\min _{\boldsymbol{U}, \boldsymbol{R}, \boldsymbol{S}, \vec{t}, j \in\left\{1,2, \ldots, N_{m}\right\}}\left(\sum_{i=1}^{N_{p}}\left\|\left(\boldsymbol{U S} \boldsymbol{R} \vec{p}_{i}+\vec{t}\right)-\vec{m}_{j}\right\|_{2}^{2}\right)
$$

$$
\begin{array}{ll}
\text { s.t. } & \boldsymbol{U}^{T} \boldsymbol{U}=I_{3}, \operatorname{det}(\boldsymbol{U})=1, \\
& \boldsymbol{R}^{T} \boldsymbol{R}=I_{3}, \operatorname{det}(\boldsymbol{R})=1, \\
& \boldsymbol{S}=\operatorname{diag}\left(s_{1}, s_{2}, \ldots, s_{m}\right), s_{j} \in\left[a_{j}, b_{j}\right]
\end{array}
$$

Where $\boldsymbol{U}$ and $\boldsymbol{R}$ are rotation matrices, $\boldsymbol{S}$ is a scale matrix.

Actually the problem can be solved by the affine ICP algorithm in the way the ICP algorithm does by iteration. At each iterative stage, two main steps are included in the affine ICP: 
[1]: establish the correspondence of the point sets with the $(k-1)$ th transformations $\left(\boldsymbol{U}_{k-1}, \boldsymbol{S}_{k-1}, \boldsymbol{R}_{k-1}, \vec{t}_{k-1}\right)$ :

$$
C_{k}(i)=\underset{j \in\left\{1,2, \ldots, N_{m}\right\}}{\arg \min }\left(\left\|\left(\boldsymbol{U}_{k-1} \boldsymbol{S}_{k-1} \boldsymbol{R}_{k-1} \vec{p}_{i}+\vec{t}_{k-1}\right)-\vec{m}_{j}\right\|_{2}^{2}\right), i=1, \ldots \ldots, N_{p}
$$

[2]: compute the $k$ th transformations $\left(\boldsymbol{U}_{k}, \boldsymbol{S}_{k}, \boldsymbol{R}_{k}, \vec{t}_{k}\right)$ :

$$
\left(\boldsymbol{U}_{k}, \boldsymbol{S}_{k}, \boldsymbol{R}_{k}, \vec{t}_{k}\right)=\underset{\boldsymbol{U}, \boldsymbol{R}, \boldsymbol{S}, \vec{t}, j \in\left\{1,2, \ldots, N_{m}\right\}}{\arg \min }\left(\sum_{i=1}^{N_{p}}\left\|\boldsymbol{U} \boldsymbol{S} \boldsymbol{R} \vec{p}_{i}+\vec{t}-\vec{m}_{C_{k}(i)}\right\|_{2}^{2}\right)
$$

\subsection{Landmarks definition for Iterative Closest Point}

The purpose of the registration technique based on the ICP mapping method is not only to align two different shapes modalities but also construct the deformation shapes according to the original shape information. The mathematical model of ICP theorem has been described above, and the method can be utilized to find out the matching points in the two corresponding shapes to generalize a new deformation shape that we can use in any further applications.

In essence ICP depends on the points that are obtained from two shapes, i.e. landmarks, the $(\mathrm{x}, \mathrm{y}, \mathrm{z})$ position points which extracted from the statistical shape from shading model(SSFS) (Figure 4.1), should be corresponding to the (x,y,z) position points which extracted from the shape from shading(SFS) (Figure 4.2).

The number of points which should be taken from each shape, depend on two things 1: taking more landmarks points from each shape, will be obtained accurate integration. 
2: Number of teeth that are part of integration process, i.e. If we have one tooth in each shape and we want to integrate between them we can get an accurate result by taking nine points .The more teeth that are part of the integration process, the number of needed points increases.

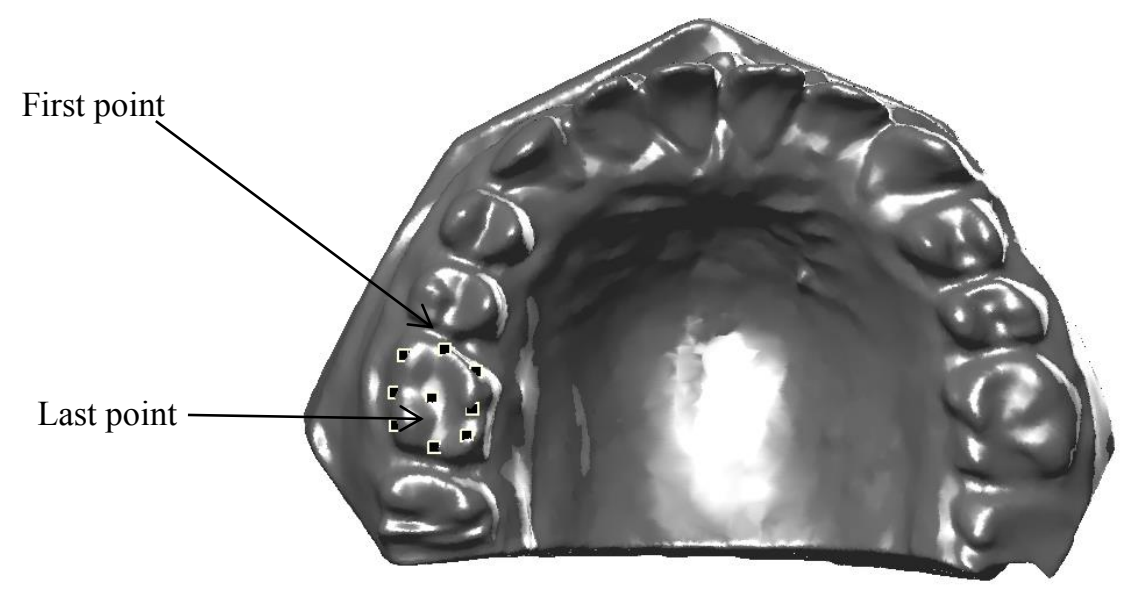

Figure 4.1. 3D-Shape of Jaw from Statistical shape from shading with the Landmarks on one tooth for registration approach. 


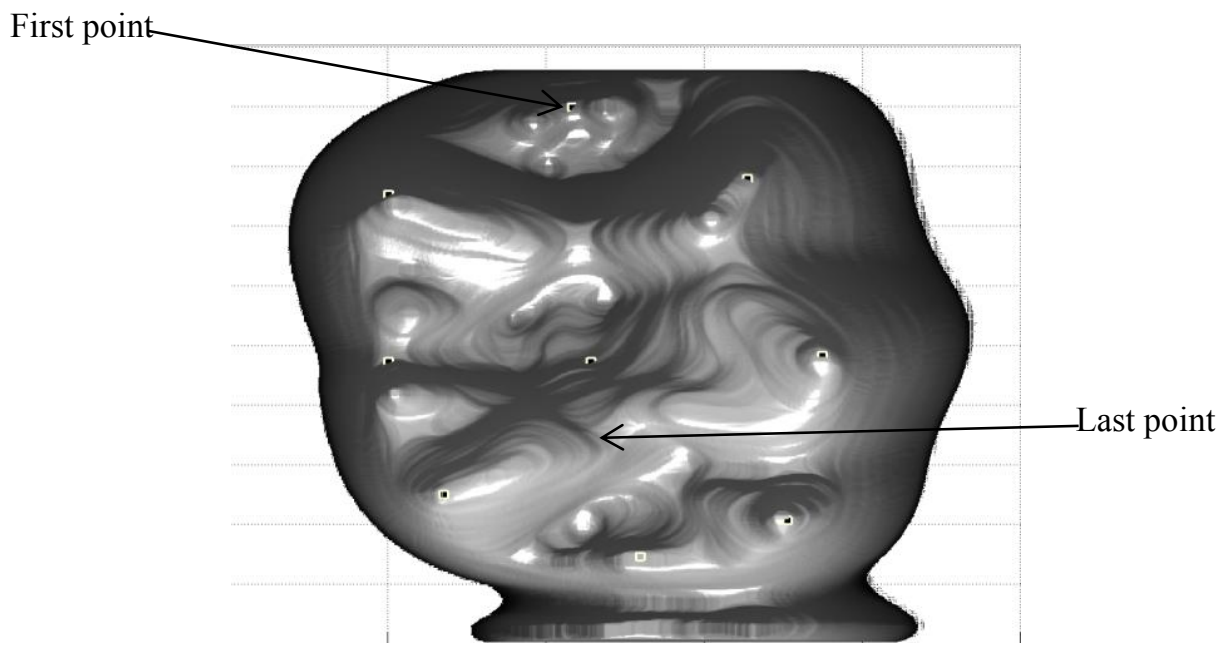

Figure 4.2. 3D-Shape of tooth from shape from shading with the Landmarks on it for registration approach

\subsection{Summary}

This chapter focused in describing the Iterative closest Point (ICP) registration for integrate between shape from shading (SFS) and statistical shape from shading (SSFS) results and then described in literary how the ICP works, finally is defined the way to choose the landmarks from these two shapes. 


\section{CHAPTER 5 \\ EXPERIMENTAL RESULTS}

This chapter will be explained in detail the result which got it from the three parts of the thesis. First in detail the result will be explained from Statistical shape from shading and compare it with the groundtruth and to monitory evaluate accuracy, Second, the shape from shading result will be explained and compare it with the groundtruth and to indicate error between them. Finally the result from statistical shape from shading and the shape from shading will be taken to integrate them by using Iterative Closest Point (ICP) for getting an accurate shape and surface result.

\subsection{D Reconstruction of Human Jaw using Statistical Shape from Shading} (SSFS)

This section shows experiments to evaluate the performance of the proposed framework in recovery 3D models for human jaws. Upper and lower jaw models are constructed from 83 upper jaw molds belonging to 45 males and 38 females with average age 20 years old (see Table 2.1 for more details). Whereas lower jaw models are constructed from 97 lower jaw molds belonging to 51 males and 46 females with average age 19 years old (see Table 2.1). There are two samples per subject, one pre-repair jaw and another post-repair jaw, referring to the jaw status before and after applying an

orthodontic teeth alignment process respectively. The statistical priors (shape, albedo and appearance models) are trained using out-of-training samples with pre- and post-repair 
instances using the oral cavity images and CT-scan of the respective molds (lower and upper jaws).

The core hypothesis of this work is that using prior information (full 3D) would enable obtaining a plausible and more accurate jaw model for the human jaw while covering regions that the classical SFS approach does not handle As such, one compares the proposed approach with a recently evaluated SFS approach [11] for tooth surface reconstruction based on the work of Ahmed and Farag in $[2,3]$. In their work, the image irradiance equation was formulated as a Partial Differential Equation (PDE) to solve for surface gradients, where the theory of viscosity solutions for Hamilton-Jacobi type equations provide a good framework of SFS algorithms [18]. Moreover, the presented work is compared with one's earlier work in [19] which assumes Lambertian reflectance and using Height maps instead of full 3D prior information.

In order to share the same metric coordinate frame, the average jaw shape $\bar{S}$ (along with its anatomical landmarks) is used as a reference to establish a dense correspondence between the groundtruth CT scan of the jaw mold corresponding to each testing image and the reconstructed shape. The alignment proceeds as follows. Procrustes-based rigid registration [21] is used to filter out translation, scale and rotation followed by $3 \mathrm{D}$ thinplate splines $[10,15]$ for non-rigid registration.

\subsubsection{Visible Crowns Reconstruction}

To evaluate the proposed approach, out-of-training jaw samples are reconstructed and compared against the ground truth CT-scan. Four types of samples are considered: 
(a)pre-repair and (b) post-repair lower jaw, (c) pre-repair and (d) post-repair upper jaw. Along with the groundtruth shapes, Figures 5.1and 5.2 show a sample of shape and albedo reconstruction of upper and lower jaws, respectively. It important to note that SFS only recovers a height map (2:5D) of the input image where there is no metric information reserved. With the metric prior used to train the offline shape model, the proposed approach reconstructs the triangular mesh (3D) corresponding to the input image. Further, most of SFS approaches assume known parameters of surface reflectance and point light source with known direction. In contrast, one's approach explicitly accounts for complex illumination and models surface reflectance without the need of the exact reflectance parameters. In Figures 5.1 and 5.2, one can observe the closeness of the SSFS-based reconstruction to the groundtruth shape. This emphasizes the role of incorporating prior-information for shape recovery as well as appearance modeling.

To evaluate the proposed approach, out-of-training jaw samples are reconstructed and compared against the groundtruth CT-scan. We assess the reconstruction accuracy in accordance with an error estimator which is based on the RMS error between the 3D points from the CT scan and the corresponding reconstructed surface points. Note that the errors are computed based on the surface points of the visible crowns to exclude the errors which might rise from the reconstruction of the mould base.

Table 5.1 reports the average and the standard deviation of the RMS error in $\mathrm{mm}$ between the 3D points from the $\mathrm{CT}$ scan and the corresponding reconstructed surface points. 
Table 5.1. Average and Std of the whole jaw surface reconstruction accuracy (RMS) in mm

\begin{tabular}{cc}
\hline \hline Jaw type & Non-Lambertian \\
\hline Upper, 12 teeth & $0.9686 \mp 0.6031$ \\
Upper, 14 teeth & $0.7873 \mp 0.3571$ \\
Lower, 12 teeth & $0.7390 \mp 0.1966$ \\
Lower, 14 teeth & $0.8195 \mp 0.3457$ \\
\hline
\end{tabular}



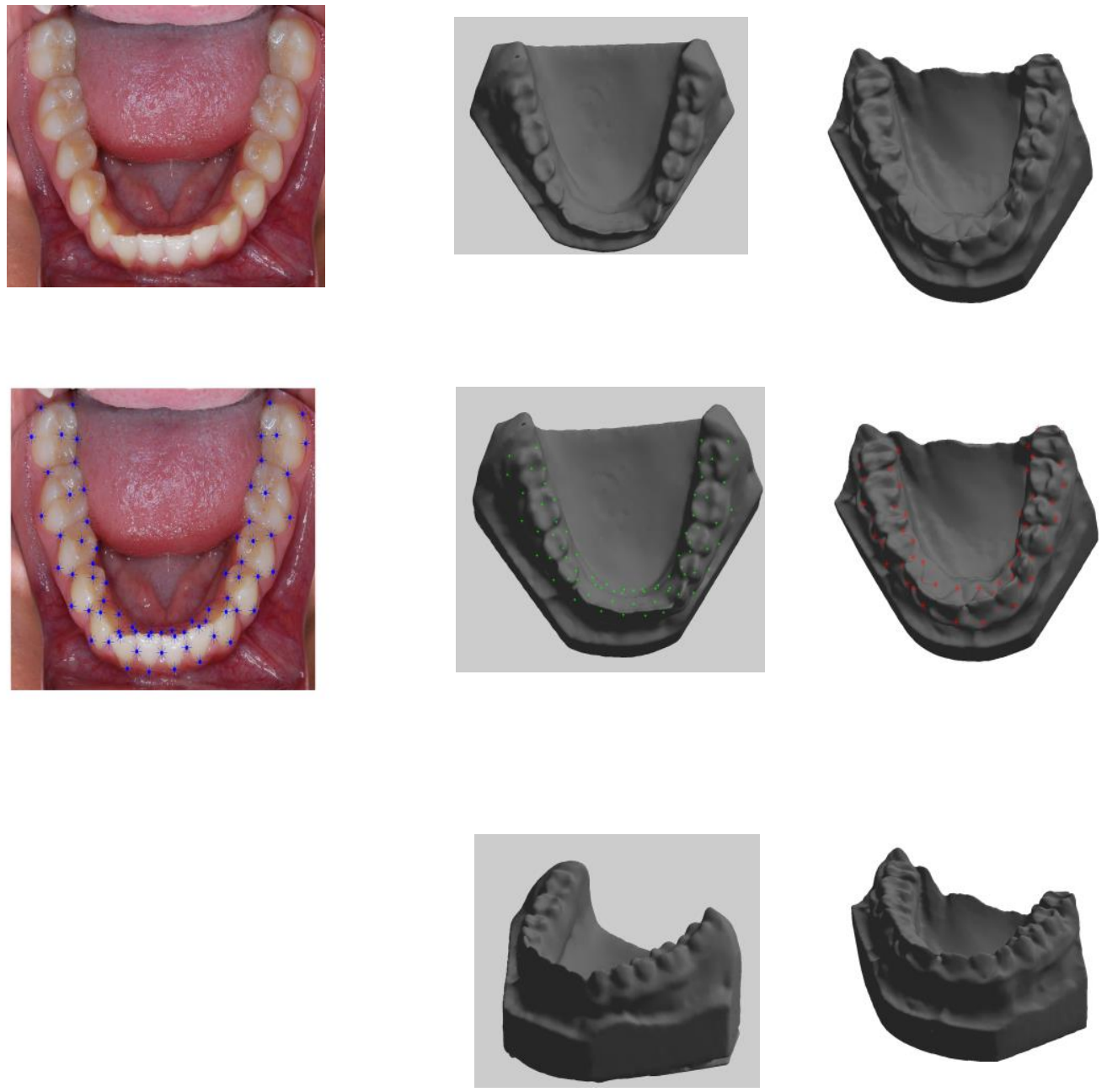

Input Image

Groundtruth

Recovered Shape

Figure 5.1. Sample reconstruction result for a Lower (post-repair) jaw( second row the Image, recovered shape and groundtruth with the landmarks of the Input Image for certain that the recovered shape take the same shape of the groundtruth). Third row show from another scene for the occlusal surface. 

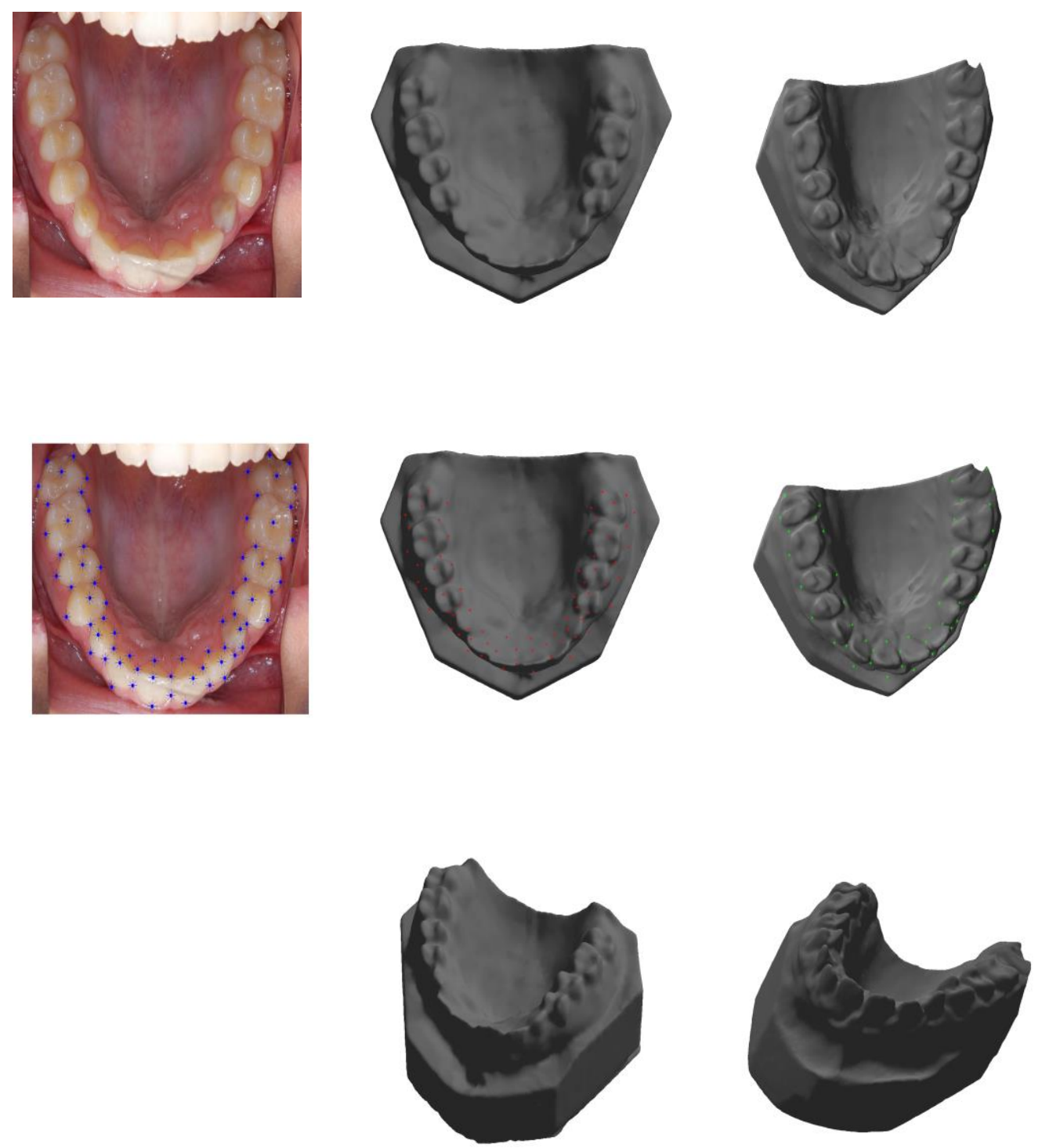

Input Image

Groundtruth

Recovered Shape

Figure 5.2. Sample reconstruction result for a Upper (post-repair) jaw( second row the Image, recovered shape and groundtruth with the landmarks of the Input Image for certain that the recovered shape take the same shape of the groundtruth).Third row show from another scene for the occlusal surface. 


\subsection{D Reconstruction of Human Teeth from a Single Image using Shape from Shading (SFS)}

A wireless setup camera is used to acquire the images of an ensemble of 84 invitro real human teeth (5 mandibular 3rd molars, 15 mandibular molars, 24 maxillary molars, 26 maxillary deciduous, and 14 premolars) while the camera is held at a distance close to the crown surface to simulate the near-imaging condition inside the human mouth. CT scanning is performed to provide more dense groundtruth information while maintaining the surface geometric detail. Figure 5.3 illustrates the 32 adult human teeth. Figure 5.4 shows samples of the acquired CT scans for one tooth type. The root part of each tooth is manually taken out from the CT scan, and the visible 3D tooth surface is segmented out.

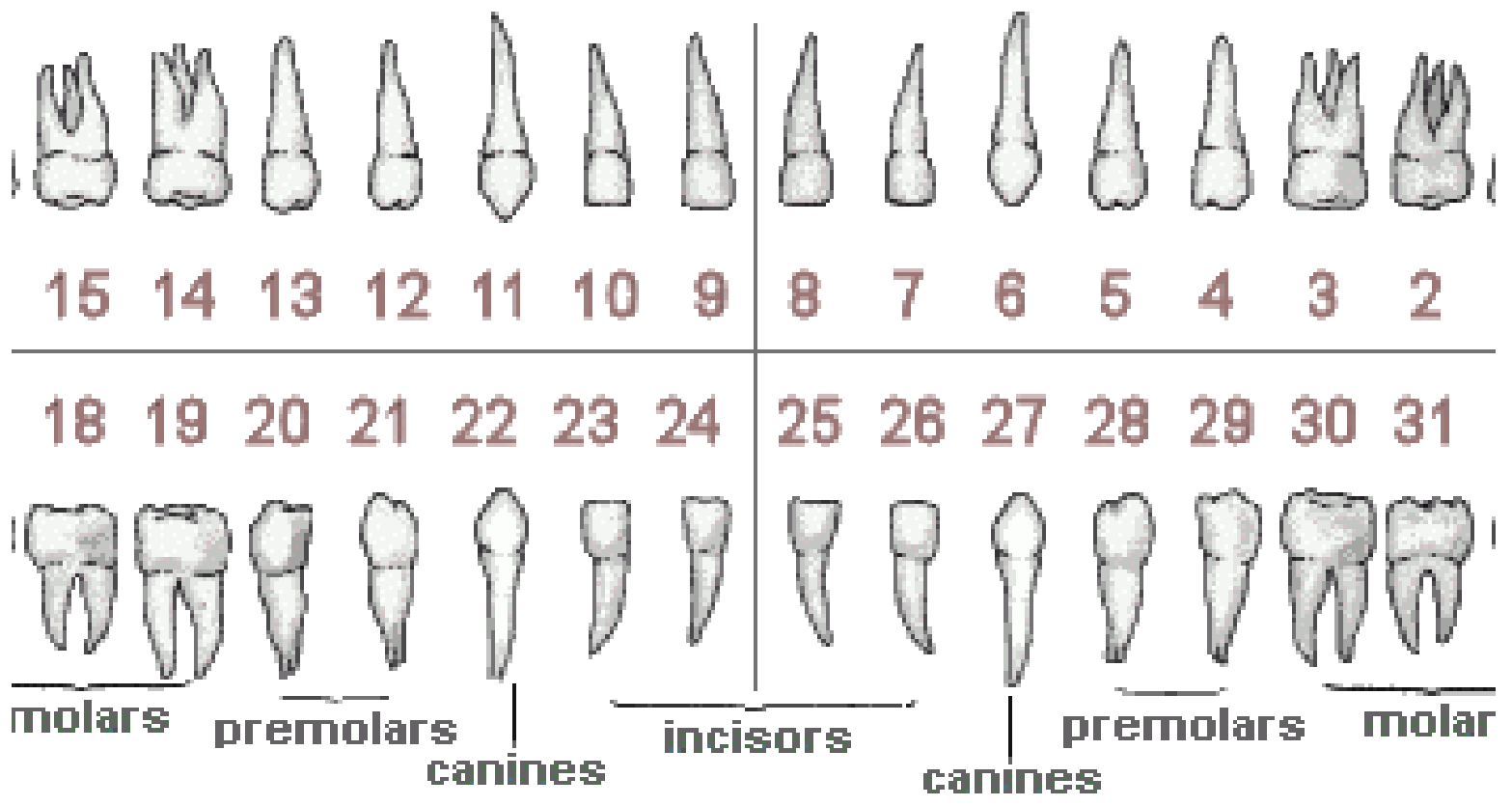

Figure 5.3. 32 Adult human teeth 


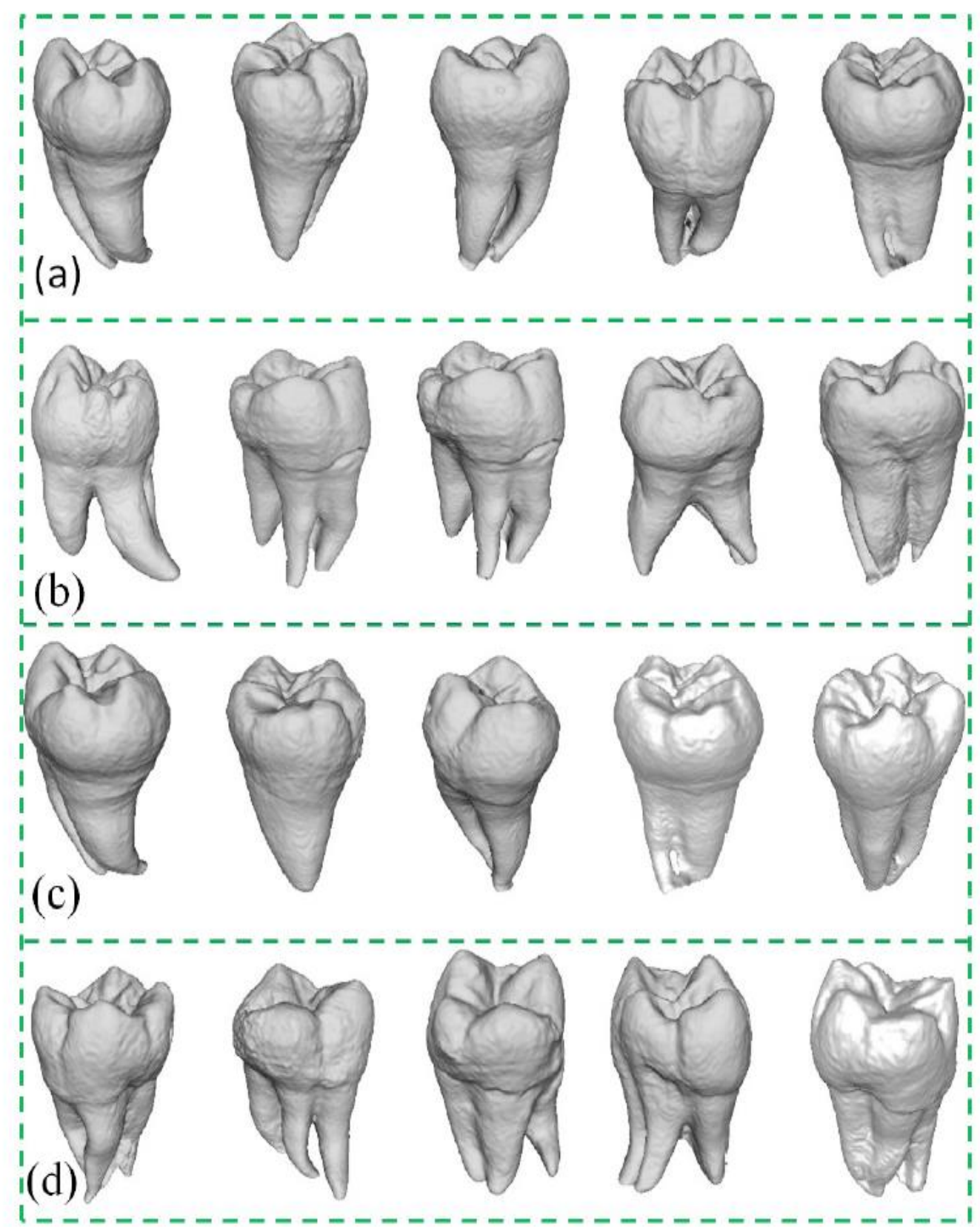

Figure 5.4. Sample CT scans for various human tooth types. (a) Maxillary deciduous teeth. (b) Maxillary molars. (c) Mandibular molars. (d) Mandibular third molars. 
Table 5.2. SFS Algorithm Panel

\begin{tabular}{|c|c|c|c|}
\hline Algorithm & Reflectance Model & Camera Parameters & Numerical Solution \\
\hline A-new & Oren-Nayar-wolff & Fully calibrated & Minimization-based \\
\hline
\end{tabular}

Table 5.3. Overall surface reconstruction accuracy (RMS) in mm

\begin{tabular}{c|c|}
\cline { 2 - 2 } Tooth type & Algorithm A-new \\
\cline { 2 - 2 } & 0.47232 \\
\hline Mandibular 3rd molars & 0.63568 \\
\hline Mandibular Molars & 0.58694 \\
\hline Maxillary Molars & 0.65997 \\
\hline Maxillary deciduous & 0.45267 \\
\hline Premolars &
\end{tabular}

Table 5.2 summarizes the proposed minimization-based solution, terms as Algorithm A.

The SFS algorithms are compared in accordance with an error estimator based on the root mean square (RMS) error between the 3D points from the CT scan and the corresponding reconstructed surface points. It is worth mentioning that throughout our experimentations. Algorithm A converges after 5-10 iterations. Table 5.3 shows the overall surface reconstruction accuracy of Algorithm A based on the testing images panel. Figure 5.4 illustrate the differences between the groundtruth (CT scanning) for different teeth and the 3D construction based on the approach (Algorithm A) 


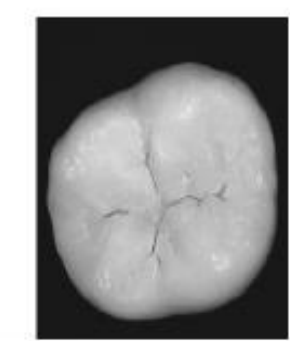

Mandibular 3rd molar

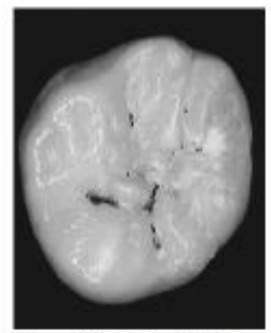

Mandibular Molar

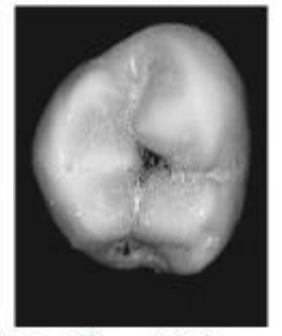

Maxillary Molars

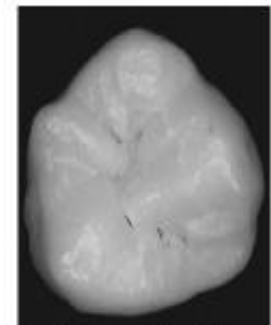

Maxillary deciduous

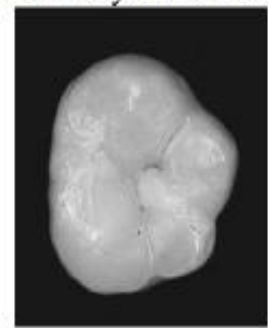

Premolars

(a)
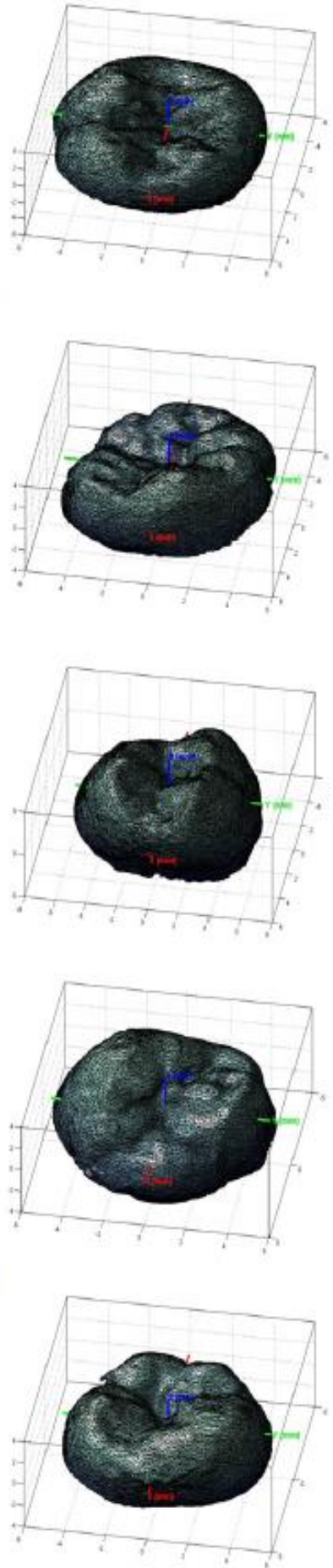

(b)

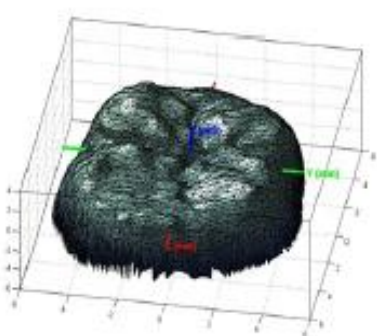

RMS $=0.43604 \mathrm{~mm}$

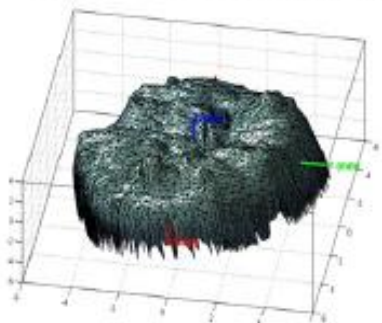

RMS $=0.47044 \mathrm{~mm}$

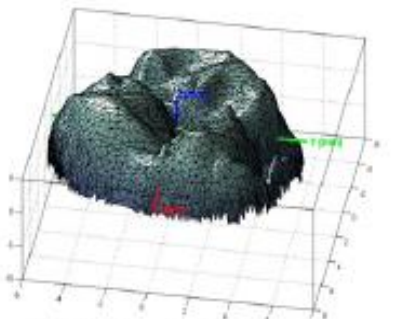

RMS $=0.66208 \mathrm{~mm}$
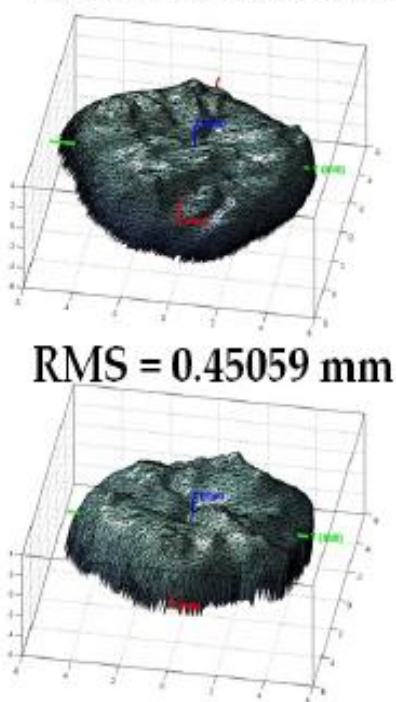

RMS $=0.36374 \mathrm{~mm}$

(c)

Figure 5.5. (a) Images for different types of human teeth, captured by the intra-oral camera. (b) Groundtruth occlusal surface generated from CT scanning. Surface reconstruction based on (c) Algorithm A 


\subsection{Iterative Closest Point Technique for 3D Shapes Registration}

This section focused on registration approach between Shape from shading and statistical shape from shading. As previously mentioned in (Chapter 4), by integration between SSFS and SFS an accurate result will be obtained and that is because the SSFS will give a shape of the jaw from just a single image but it does not give a truly surface for the tooth so the SSFS was supported by SFS approach.

SSFS was used for the situations where the SFS cannot handle . the SFS approach if it is used for one tooth it will give an accurate result comparing with groundtruth because it need near illumination, but if it is used of a whole jaw it won't give a result like SSFS give (see Figure 5.7).

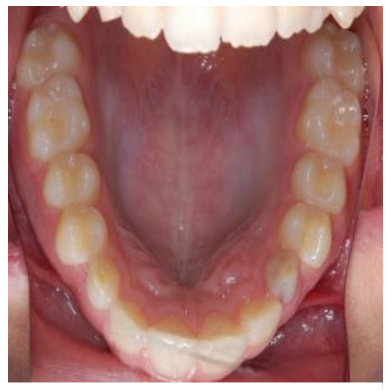

Input Image

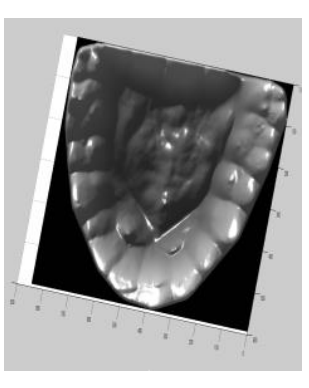

3D construction (SFS)

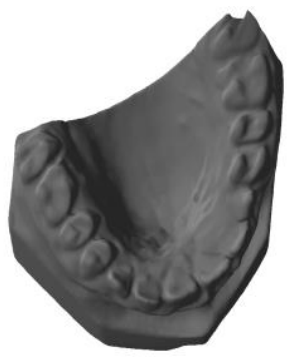

3D construction (SSFS)

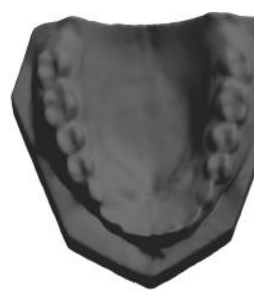

Groundtruth (CT scanning)

Figure 5.6. The Input Image and the difference between the SSFS and SFS approach in $3 \mathrm{D}$ construction for a jaw and comparing them with groundtruth (CT scanning) 
As following, we will move to a practical part and illustrate the TPS approach in steps.

The single image will be taken and make a manual annotation for it if the jaw contain 14 teeth , 72 Landmarks will be obtained but if it contain 12 teeth just 62 Landmarks ,the experimental will be 14 teeth, around each tooth and in the center of it there is 7 Landmarks (Figure 5.7)

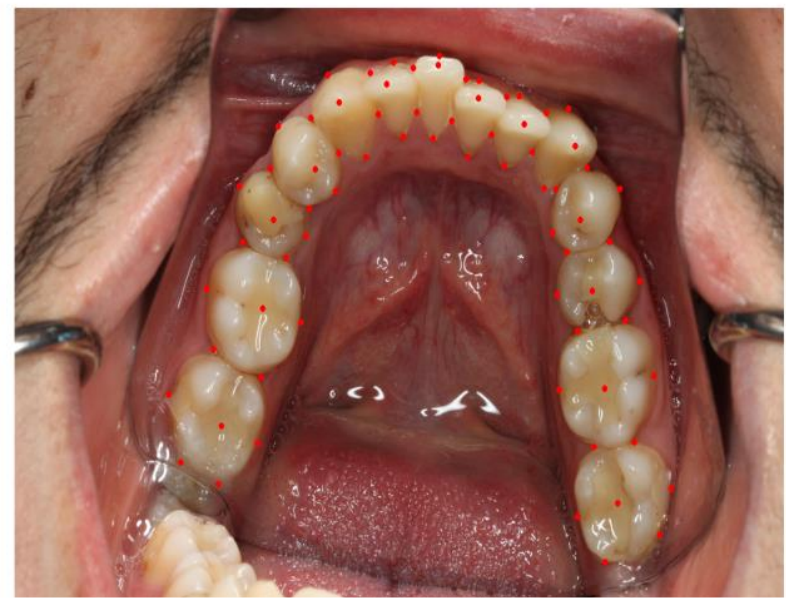

Figure 5.7. Input Image with the Landmarks (Landmarks illustrate in red color points)

Then this jaw will be taken and build the 3D scene by Statistical shape from shading (Figure 5.8). Before the 3D processing the characteristic of the input should be determined, if is it pre repair or post repair and is it contain 14 teeth or 12 teeth and is it upper or lower, this experiment contains 14 teeth and jaw type lower and status jaw pre repair. 

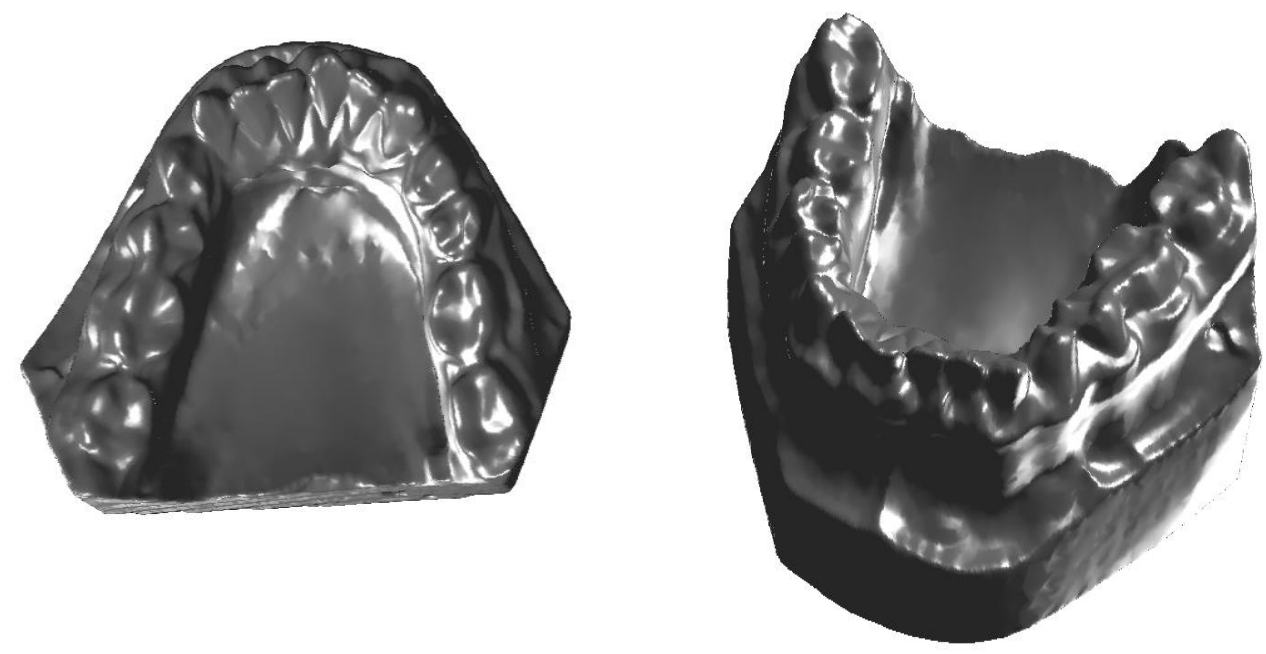

Figure 5.8. Show the 3D construction for the Input Image (Figure 5.7) jaw with a different scene

Also an algorithm was developed to recovered the albedo from the input jaw image and interpolate it with the 3D shape of the jaw (Figure 5.9)

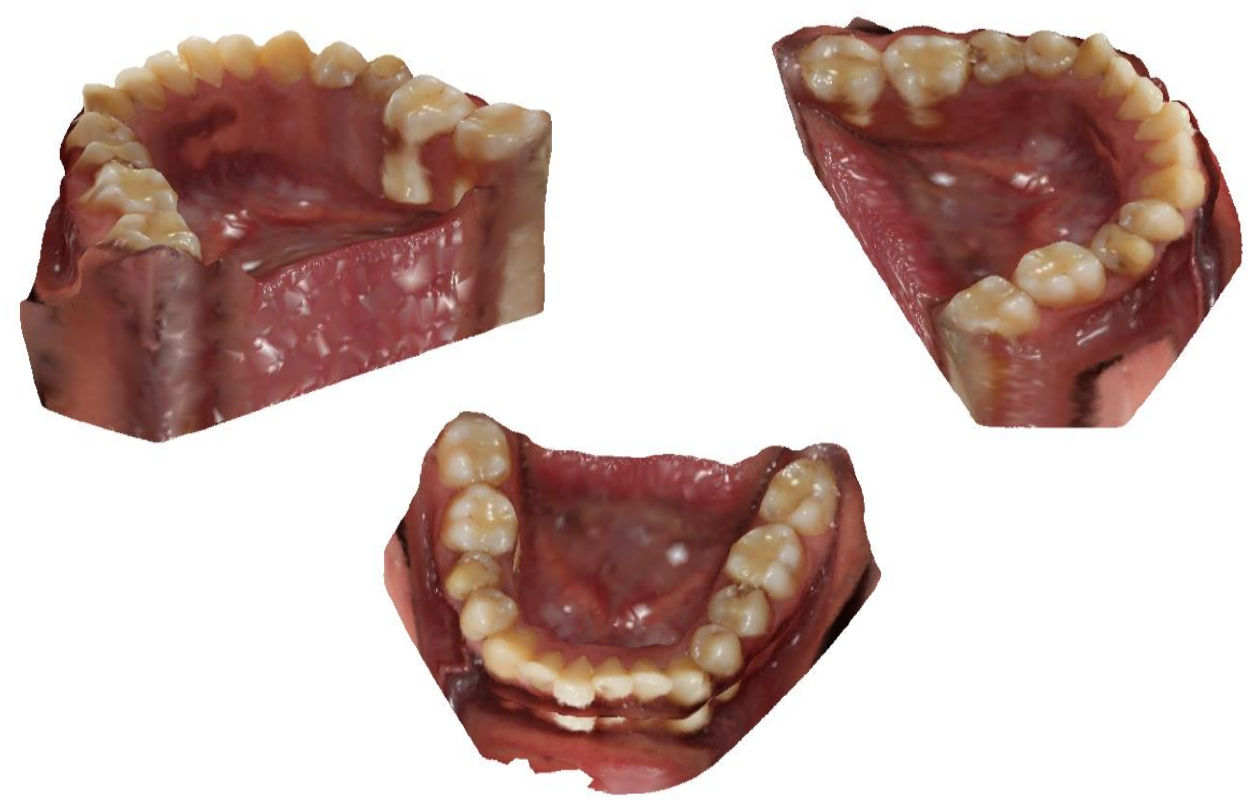

Figure 5.9. 3D- shape for the jaw with the albedo recovered 
Now the tooth from this jaw will be taken (Figure 5.10) and the 3D shape will be constructed by Shape from shading algorithm (Figure 5.11)

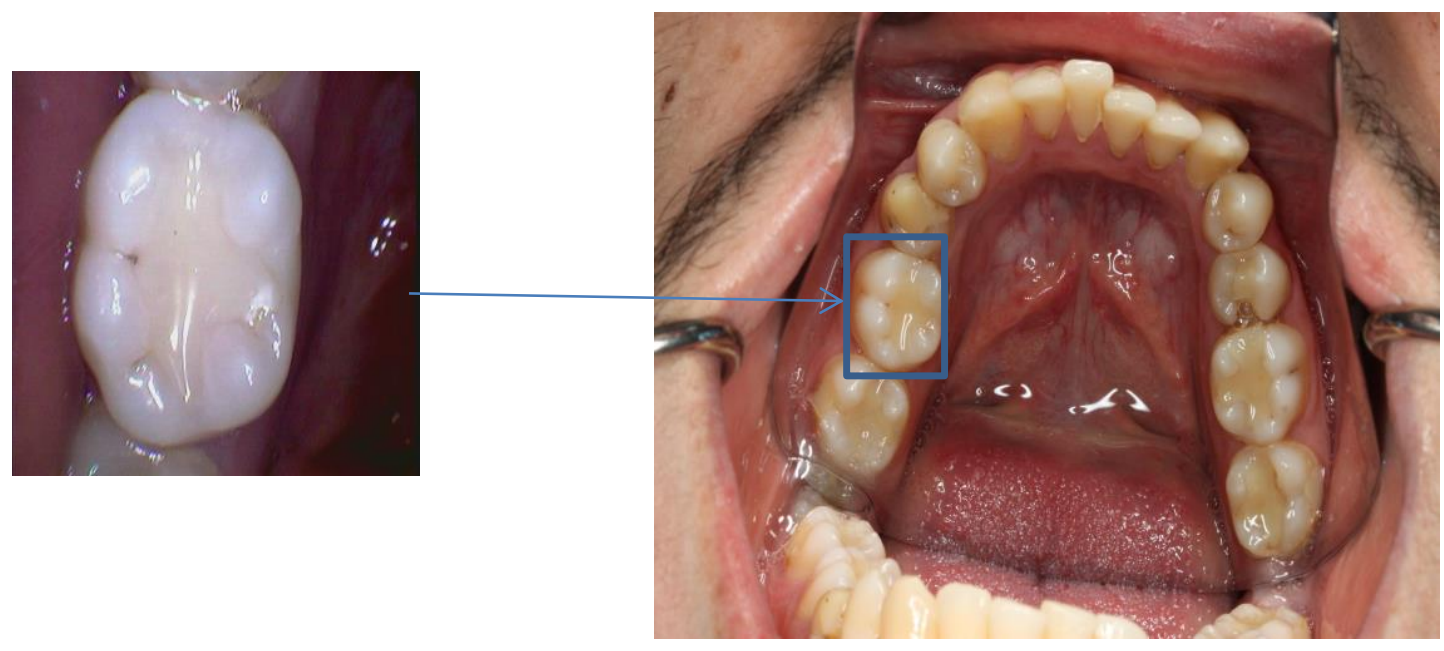

Figure 5.10. Input tooth Image
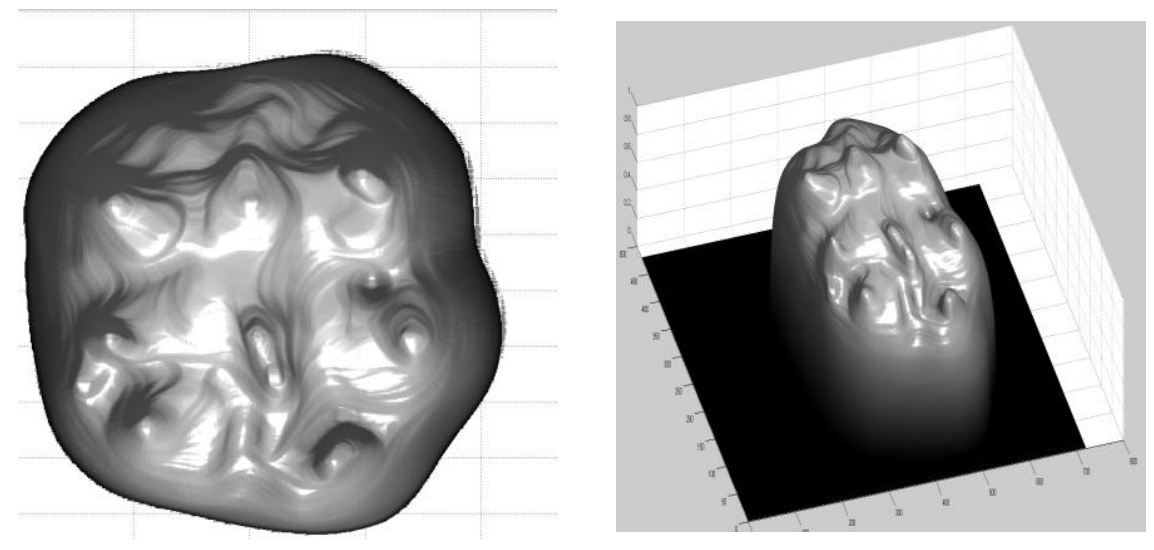

Figure 5.11. 3D reconstructions with different scenes for the tooth Image from shape from shading 
After the 3D shapes were obtained for both input jaw and one tooth for this jaw, the Landmarks will be taken from 3D shape jaw (Figure 5.12). As previously mentioned in (Chapter 4) if the integration is between just two teeth, must be take at least nine points to get accurate results.

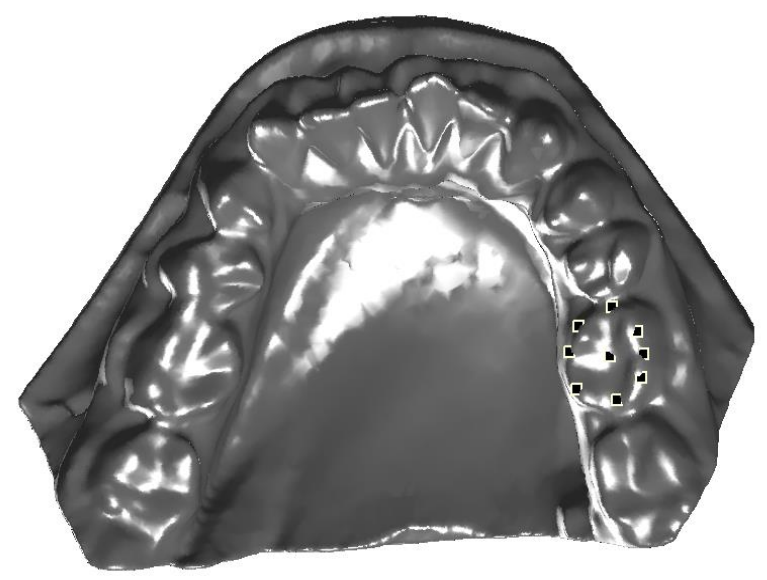

Figure 5.12. 3D shape for the jaw with the Landmarks

Now the Landmarks from 3D shape tooth will be taken jaw (Figure 5.13).

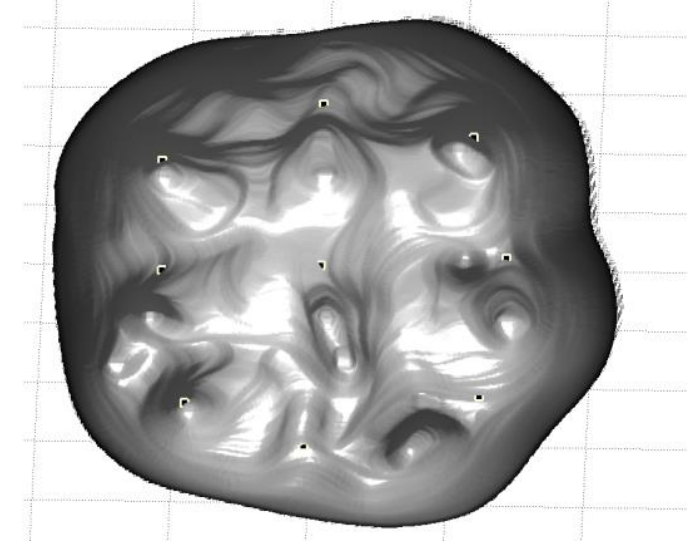

Figure 5.13. 3D shape tooth with the Landmarks 
After getting the landmarks for the 3D shape tooth and the correspondence landmarks from the 3D shape jaw, applying these points on the Iterative Closest Point (ICP) algorithm to obtain the deformation equation. Finally after obtained the ICP equation, the 3D shape tooth was applied to become in the same domain of the 3D shape jaw (Figure 5.14) 

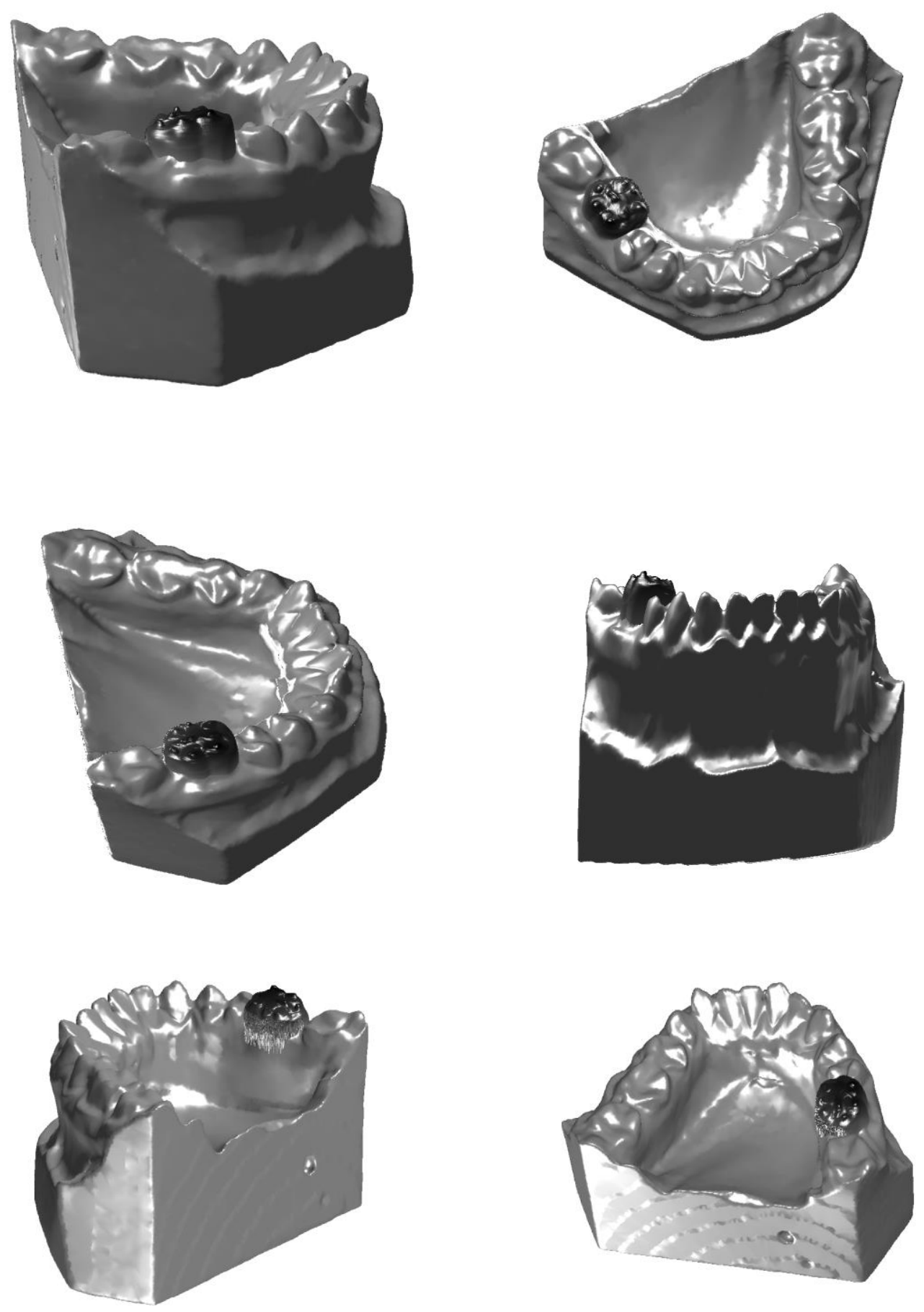

Figure 5.14. 3D shape with different scenes obtained from the Integration approach between the statistical shape from shading (SSFS) and shape from shading (SFS). 


\subsection{Graphic User Interface Approach for Jaw Construction Shape}

After completing this approach, GUI (Graphic User Interface) was built for the Jaw construction shape (Figure 5.15).

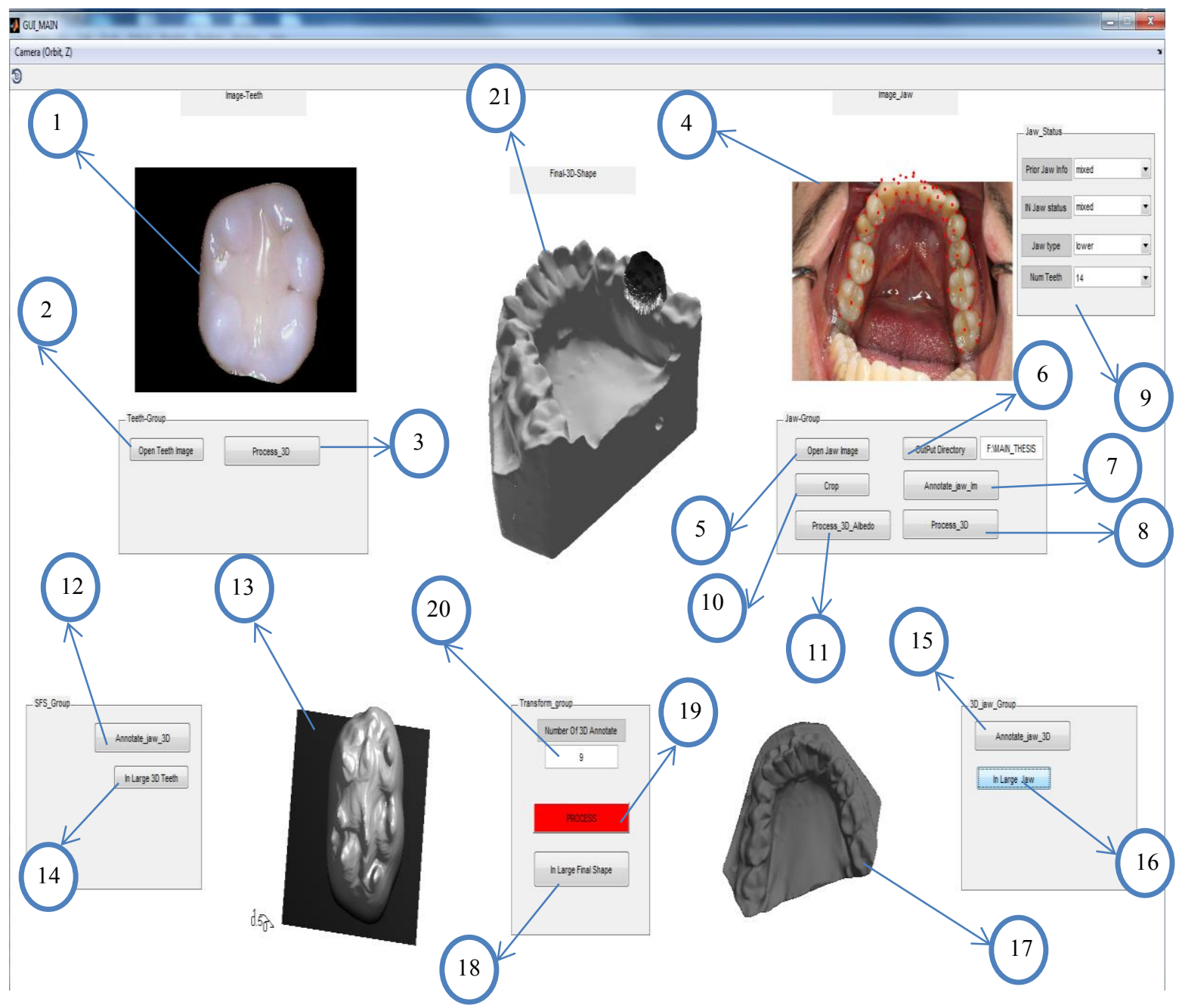

Figure 5.15. Graphic User Interface Approach for Jaw Construction Shape 
1-Tooth Image Axes: hold Input tooth image.

2-Open tooth Image: Select Input tooth Image for shape from shading approach.

3-Process-3D: hold the input tooth image and apply (SFS) algorithm on it.

4- Jaw Image Axes: hold Input jaw image.

5-Open Jaw Image: Select Input jaw Image for statistical shape from shading approach.

6-Output Directory: Determine folder for saving the output from the approach on it.

7-Annotate-jaw-im: Select the Landmarks for the Input jaw Image (annotation process).

8- Process-3D: hold the input jaw image and apply (SSFS) algorithm on it.

9-Determine the characteristic of Input Jaw:

A: Prior jaw info: Determine status of jaw.

B: IN jaw status: Determine the actual status of jaw.

C: Jaw type: Determine the input jaw type (lower, upper).

D: Num Teeth: Determine the number of teeth in the input jaw $(12,14)$.

10-Crop: making a shrink for the input jaw image if it need.

11-Process_3D_Albedo: recovered the albedo from the input image jaw.

12- Annotate-jaw-3D: Select the Landmarks for the 3D shape of tooth.

13- Tooth Shape Axes: hold Input tooth 3D shape.

14-In Large 3D Teeth: Open the 3D shape tooth in new figure.

15- Annotate-jaw-3D: Select the Landmarks for the 3D shape of jaw.

16- In Large jaw: Open the 3D shape jaw in new figure.

17- Jaw Shape Axes: hold jaw 3D shape.

18- In Large Final Shape: Open the 3D shape after the integration process in new figure. 19-Number of 3D annotate: Determine the number of Landmarks for 3D annotate.

20-PROCESS: run Thin-Plate Spline algorithm between the tooth and jaw.

21- Final-3D-Shape Axes: hold the output 3D shape from TPS algorithm. 


\subsection{Summary}

this chapter focused on showing the experimental and result part of the approach, since at the beginning the Statistical shape from shading part was explained in detail and supported it with pictures for the output and then starting to show the result from shape from shading part and also support it by pictures, then the TPS registration algorithm was explained by taking the result from shape from shading part and statistical shape from shading part for enhance the result. Finally GUI was built for combining the three main parts of this approach. 


\section{CHAPTER 6 CONCLUSIONS AND FUTURE WORK}

This thesis has presented 3D reconstruction and shape modeling with applications to dentistry.

\subsection{Summary of Contributions}

The findings from this thesis make several contributions to the current literature which can be summarized as follows:

1. In Chapter 2, a model-based SFS approach has been proposed which allows for the construction of plausible human jaw models in vivo, without ionizing radiation, using fewer sample points in order to reduce the cost and intrusiveness of acquiring models of patients; teeth/jaws over time.

2. Chapter 3 focused on the 3D surface reconstruction aspect for human teeth based on a single image. A more realistic formulation of the SFS was introduced problem by considering the image formation components; the camera, the light source, and the surface reflectance. Proposed a non-Lambertian SFS algorithm under perspective projection which benefits from camera calibration parameters. We take into account the attenuation of illumination due to near-field imaging. 
3. In Chapter 4, a convenient 3D shape registration technique based on Iterative Closest Point (ICP) mapping function was presented. The method can determine the corresponding 3D shape information and obtain the matching shape structure from different shapes

\subsection{Future work}

1. The presented approach throughout this work uses manual annotation which increases the overall time of the $3 \mathrm{D}$ reconstruction process. In the future, it is intended to investigate using computer vision methods for automating the annotation process.

2. The proposed works only if the jaw contains 12 teeth or 14 teeth. In the future, it could be extended to work with any number of teeth. That could be achieved by making an automatic segmentation approach for the teeth. 


\section{REFERENCES}

[1] A. S. Abdelrahim, M. A. Abdelrahman, H. Abdelmunim, A. Farag, and M. Miller. Novel image-based $3 \mathrm{~d}$ reconstruction of the human jaw using shape from shading and feature descriptors. In Proceedings of the British Machine Vision Conference, pages 41.1-41.11. BMVA Press, 2011.

[2] A.H. Abdelrehim and A. A. Farag. A new formulation for shape from shading for non lambertian surfaces. In International Conference on Computer Vision and Pattern Recognition CVPR06, pages 1817-1824, NY, USA, 2006.

[3] A. Ahmed and A. Farag. Shape from shading under various imaging conditions. In Proc. of IEEE Conference on Computer Vision and Pattern Recognition (CVPR'07), Minneapolis, MN, pages X1-X8, June 18-23, 2007.

[4] A.H. Ahmed and A. A. Farag. A new statistical model combining shape and spherical harmonics illumination for face reconstruction. In Proc. ISVC, pages 531541, 2007.

[5] J. Atick, P. Griffin, and A. Redlich. Statistical approach to shape from shading: Reconstruction of 3D face surfaces from single 2D images. Neural Computation, 8:1321-1340, 1997.

[6] R. Basri, D. Jacobs, and I. Kemelmacher. Photometric stereo with general, unknown lighting. International Journal of Computer Vision, 72[3]:239-257, 2007.

[7] R. Basri and D. W. Jacobs. Lambertian reflectance and linear subspaces. IEEE Transactions on Pattern Analysis and Machine Intelligence, 25[2]:218-233, Feb 2003.

[8] V. Blanz, A. Mehl, T. Vetter, and H. Seidel. A statistical method for robust 3d surface reconstruction from sparse data. In In International Symposium on 3D Data Processing, Visualization and Transmission, pages 293-300, 2004. 
[9] Volker Blanz and Thomas Vetter. Face recognition based on fitting a 3d morphable model. IEEE Trans. Pattern Anal. Mach. Intell., 25[9]:1063-1074, 2003.

[10] F. Bookstein. Principal warps: Thin-plate splines and the decomposition of deformations. IEEE Transactions on Pattern Analysis and Machine Intelligence, 11:567-585, 1989.

[11] C.N. Carter, R.J. Pusateri, Dongqing Chen, A.H. Ahmed, and A.A. Farag. Shape from shading for hybrid surfaces as applied to tooth reconstruction. In Image Processing (ICIP), 2010 17th IEEE International Conference on, pages 4049-4052, 2010.

[12] M. Castelan and J. Van Horebeek. Relating intensities with three dimensional facial shape using partial least squares. Computer Vision, IET, 3:60-73, June 2009.

[13] M. Castelan, W. Smith, and E. Hancock. A coupled statistical model for face shape recovery from brightness images. IEEE Transaction on Image Processing, 16:11391151, 2007.

[14] European Commission. European guidelines on radiation protection in dental radiology. Radiation Protection Issue number 136, 2004.

[15] T. Cootes and C. Taylor. Statistical models of appearance for computer vision. Technical report, Imaging Science and Biomedical Engineering, University of Manchester, Manchester M13 9PT, U.K., March 2004.

[16] Timothy F. Cootes, Gareth J. Edwards, and Christopher J. Taylor. Active appearance models. IEEE Transactions on Pattern Analysis and Machine Intelligence, 23:681-685, 2001.

[17] R. Dovgard and R. Basri. Statistical Symmetric Shape from Shading for 3D Structure Recovery of Faces. In Proceedings of the 8th European Conference on Computer Vision (volume II), 3022 of Lecture Notes in Computer Science, pages 99-113, Prague, Czech Republic, May 2004. 
[18] J. D. Durou, M. Falcone, and M. Sagona. Numerical methods for shape fromshading: A new survey with benchmarks. Computer Vision and Image Understanding, 109[1]:22 - 43, 2008.

[19] A. Farag, S. Elhabian, A. Abdelrahim, W. Aboelmaaty, A. Farman, and D. Tazman. Model-based human teeth shape recovery from a single optical image with unknown illumination. In MICCAI Medical Computer Vision Workshop (MCV), 2012.

[20] A. A. Goshtasby, S. Nambala, W. G. deRijk, and S. D. Campbell. A system for digital reconstruction of gypsum dental casts,. In IEEE Transactions on Medical Imaging, 16, pages 664-674, Oct 1997.

[21] J. Gower. Generalized procrustes analysis. Psychometrika, 40[1]:33-51, 1975.

[22] A. Hallquis, L. Hardell, A. Degerman, G. Wingren, and L. Boquist. Medical diagnostic and therapeutic ionizing radiation and the risk for thyroid cancer: a case-control study. Eur J Cancer Prevention 3:259-67, 1994.

[23] B. Horn. Robot Vision. McGraw-Hill, 1986.

[24] B. K. P. Horn and M. J. Brooks. The variational approach to shape from shading. Comput. Vision Graph. Image Process., 33:174\{208, February 1986.

[25] B.K.P. Horn. Shape from Shading: A Method for Obtaining the Shape of a Smooth Opaque Object from One View. PhD thesis, Massachusetts Inst. of Technology, Cambridge, Massachusetts, 1970.

[26] P. L. Horn-Ross, B. M. Ljung, and M. Morrow. Environmental factors and the risk of salivary gland cancer. Epidemiology 8:414-9, 1997.

[27] S. K. Nayar L. B.Wolff and M. Oren. Improved diffuse reflection models for computer vision. IJCV, 30[1]:55-71, 1998.

[28] C. Thode Larsen. Modelling and Rendering of Teeth - A Study in Sub Surface Scattering. Academic dissertation (Master thesis), Technical University of Denmark (DTU), Kgs. Lyngby, Denmark, 2011.

[29] Rich (CRT) Marino. Dental anatomy: Quick study academic, 2004. Barcharts Inc, illustrated by: Vincent Perez. 
[30] S. Milborrow and F. Nicolls. Locating facial features with an extended active shape model. ECCV, 2008.

[31] H. L. Mitchell and R. G. Chadwick. Challenges of photogrammetric intraoral tooth measurement. In The International Archives of the Photogrammetry, Remote Sensing and Spatial Information Sciences, Kyoto, Japan,, pages 779-782, 2008.

[32] M. Oren and S. Nayar. Generalization of lambert's reflectance model. In Computer Graphics, 28(Annual Conference Series), pages 239-246, 1994.

[33] E. Prados, F. Camilli, and O. Faugeras. A unifying and rigorous shape from shading method adapted to realistic data and applications. Journal of Mathematical Imaging and Vision, 25:307-328, 2006.

[34] E. Prados, F. Camilli, and O. Faugeras. A unifying and rigorous shape from shading method adapted to realistic data and applications. J. Math. Imaging Vis., 25:307-328, 2006.

[35] E. Prados and O. Faugeras. Shape from shading: a well-posed problem? In International Conference on Computer Vision and Pattern Recognition CVPR05, San Diego, CA, USA, June 2005.

[36] J. Ragain and W. Johnston. Accuracy of kubelka-munk reectance theory applied to human dentin and enamel. Journal of Dental Research, 80[2]:449-452, 2001.

[37] Hossein Ragheb and Edwin R. Hancock. Surface radiance correction for shape from shading. Pattern Recognition, 38:1574-1595, 2005.

[38] H. Rara, S. Elhabian, T. Starr, and A. Farag. Model-based shape recovery from single images of general and unknown lighting. In 16th IEEE International Conference on Image Processing (ICIP2009), pages 517-520, 7-10 Nov. 2009.

[39] H. Rara, S. Elhabian, T. Starr, and A. Farag. 3d face recovery from intensities of general and unknown lighting using partial least squares. In $17^{\text {th }}$ IEEE International Conference on Image Processing (ICIP2010), Sept. 2010.

[40] W. A. P. Smith and E. R. Hancock. Recovering facial shape using a statistical model of surface normal direction. IEEE Trans. on Pattern Analysis and Machine Intelligence, 28. 
[41] K. Wilson, N. Sun, M. Huang, W. Zhang, A. Lee, A. Li, S. Wang, and J. Wu. E_ects of ionizing radiation on self renewal and pluripotency of human embryonic stem cells. Cancer Res. 70(13): 55395548, 2010.

[42] S. M. Yamany, A. A. Farag, D. Tasman, and A. G. Farman. A 3-d reconstruction system for the human jaw using a sequence of optical images. IEEE Transactions on Medical Imaging, 19:538-547, May 2000.

[43] L. Zhang and D. Samaras. Face recognition from a single training image under arbitrary unknown lighting using spherical harmonics. IEEE Transaction on Pattern Analysis Machine Intelligence, 28:351-363, 2006.

[44] R. Zhang, P. Tsai, J. E. Cryer, and M. Shah. Shape from shading: A survey. IEEE Transactions on Pattern Analysis and Machine Intelligence, 21[1]:690- 706, 1999.

[45] Wen Yi Zhao and Rama Chellappa. Symmetric shape-from-shading using selfratio image. Int. J. Comput. Vision, 45[1]:55-75, 2001.

[46] J. Zijp and J. Rudolf. Optical properties of dental hard tissues. Dissertations, University of Groningen, 2001.

[47] E. Prados, O. Faugeras, and F. Camilli. Shape from shading: a well-posed problem? RR 5297, INRIA Research, August 2004.

[48] H. Rara, S. Elhabian, T. Starr, and A. Farag. A statistical model combining shape and spherical harmonics for face reconstruction and recognition. In Biomedical Engineering Conference, CIBEC2008. Cairo International, pages 1-4, 18-20 Dec. 2008.

[49] Shaoyi Du, Nanning Zheng, Shihui Ying, Jianyi Liu . Affine iterative closest point algorithm for point set registration. Pattern Recognition Letters 31 (2010) 791-799.

[50] Du, S.Y., Zheng, N.N., Ying, S.H., You, Q.B., Wu, Y., 2007. An extension of the ICP algorithm considering scale factor. In: Proc. 14th IEEE Internat. Conf.on Image Processing (ICIP), pp. 193-196.

[51] Amberg, B., Romdhani, S., Vetter, T., 2007. Optimal step nonrigid ICP algorithms for surface registration. In: Proc. IEEE Conf. on Computer Vision and Pattern Recognition (CVPR), pp. 1-8. 
[52] Jian, B., Vemuri, B.C., 2005. A Robust algorithm for point set registration using mixture of Gaussians. In: Proc. IEEE Internat. Conf. on Computer Vision (ICCV), pp. 1246-1251.

[53] Chui, H., Rangarajan, A., Zhang, J., Leonard, C.M., 2004. Unsupervised learning of an atlas from unlabeled point-sets. IEEE Pattern Anal. Machine Intell. 26 (2), $160-172$.

[54] Wang, F., Vemuri, B.C., Rangarajan, A., Eisenschenk, S.J., 2008. Simultaneous nonrigid registration of multiple point sets and atlas construction. IEEE Trans. Pattern Anal. Machine Intell. 30 (11), 2011-2022.

[55] Feldmar, J., Ayache, N., 1996. Rigid, affine and locally affine registration of free-form surfaces. Internat. J. Comput. Vision 18 (2), 99119.

[56] Ho, J., Yang, M., Rangarajan, A., Vemuri, B., 2007. A new affine registration algorithm for matching 2D point sets. In: Proc. IEEE Workshop on Applications of Computer Vision (WACV), pp. 25-30.

[57] Chui, H., Rangarajan, A., 2003. A new point matching algorithm for non-rigid registration. Computer Vision and Image Understanding 89 (2-3), 114-141.

[58] McNeill, G., Vijayakumar, S., 2006. Part-based Probabilistic Point Matching. In: Proc. 18th Internat. Conf. on Pattern Recognition (ICPR), pp. 382-386.

[59] Megyesi, Z., Kos, G., Chetverikov, D., 2006. Dense 3D reconstruction from images by normal aided matching. Machine Graphics Vision 15 (1), 3-28.

[60] Myronenko, A., Song, X., Carreira-Perpinan, M., 2006. Non-rigid point set registration: Coherent Point Drift. In: Proc. Advances in Neural Information Processing Systems (NIPS), pp. 1009-1016.

[61] Besl, P.J., McKay, H.D., 1992. A method for registration of 3-D shapes. IEEE Trans.Pattern Anal. Machine Intell. 14 (2), 239-256. 


\title{
CURRICULUM VITA
}

\section{NAME:}

Mohamad Ghanoum

\author{
ADDRESS: \\ Department of Electrical Engineering \\ 2211 South Brook \\ Lutz Hall, Room \#6, Louisville, KY 40292, USA \\ University of Louisville
}

\section{DOB:}

Damas Suburb, Syria - Jan 28, 1990

\section{OBJECTIVE:}

Pursuit of advanced research and development in computer vision, image processing, and medical imaging as a postdoctoral fellow.

\section{RESEARCH INTEREST:}

My research/development interests lie in the fields of computer vision, image understanding and pattern recognition. My primary focus is 3D shape reconstruction, shape-from-X, statistical shape analysis, and medical imaging. 


\section{EDUCATION:}

Master of Science in Electrical and Computer Engineering,

University of Louisville, Louisville, Kentucky, USA

2014-15

- Thesis Topic: 3D-Reconstruction of Human Jaw from a Single Image:

Integration between Shape from Shading and Statistical Shape from Shading.

- GPA: 3.708 out of 4

- Advisor: Professor Aly A. Farag

- Area of Study: Computer Vision and Image Processing

Bachelor Degree, Communication and Electronic Engineering

University of Kalamoon, Syria

2008-2013

- Thesis Topic: GSM IMULATION USING TDMA TECHNOLOGY.

- GPA: 3.57 out of 4

- Advisor: Dr. Atalla Sayda

- Area of Study: Communication System

\section{TRAINING:}

-Multivariate Data Analysis , Madrid ,Spain/Polytichnical University of Madrid/2009

-Data Mining, Madrid ,Spain/Polytichnical University of Madrid/2009

-CCNA(Cisco certified Network associate),200-120,version 2,Hyderabad, India /2013 
-CCNA Security(Cisco certified Network Security),Hyderabad, India/2013

-CCNA Data Center(Cisco Certified Associate Data Center) Hyderabad, India /2013

-Certified Penetration Testing Professional (CPTP), Hyderabad, India /2013

- CCNA Voice(Cisco Certified Network Associate Voice) ,Hyderabad, India /2013

- CCNP Voice(Cisco Certified Network Professional Voice),Hyderabad, India /2013

- CCNP Security(Cisco Certified Professional Security),Hyderabad, India /2013

\section{SKILLS:}

Programming Languages

- Matlab, Professional

- C, Fair

\section{ORGANIZATION:}

- IEEE (Institute Of Electrical and Electronics Engineers)

- Golden Key International Honor Society

\section{HONORS and AWARDS:}

- First rank on top of my graduating class, Bachelor degree, University Of Kalamoon.

- Scholarship from University of Louisville Graduate School, Master degree. 